\title{
Powers of the Euler product and commutative subalgebras of a complex simple Lie algebra
}

\author{
BERTRAM KOSTANT*
}

\begin{abstract}
If $\mathfrak{g}$ is a complex simple Lie algebra, and $k$ does not exceed the dual Coxeter number of $\mathfrak{g}$, then the $\mathrm{k}^{\text {th }}$ coefficient of the $\operatorname{dim} \mathfrak{g}$ power of the Euler product may be given by the dimension of a subspace of $\wedge^{k} \mathfrak{g}$ defined by all abelian subalgebras of $\mathfrak{g}$ of dimension $k$. This has implications for all the coefficients of all the powers of the Euler product. Involved in the main results are Dale Peterson's $2^{\text {rank }}$ theorem on the number of abelian ideals in a Borel subalgebra of $\mathfrak{g}$, an element of type $\rho$ and my heat kernel formulation of Macdonald's $\eta$-function theorem, a set $D_{\text {alcove }}$ of special highest weights parameterized by all the alcoves in a Weyl chamber (generalizing Young diagrams of null $m$-core when $\mathfrak{g}=\operatorname{Lie} \operatorname{Sl}(m, \mathbb{C})$ ), and the homology and cohomology of the nil radical of the standard maximal parabolic subalgebra of the affine Kac-Moody Lie algebra.
\end{abstract}

\section{Introduction}

0.1. The Euler product is the power series in the variable $x$ obtained from the expansion of the infinite product $\Pi_{n=1}^{\infty}\left(1-x^{n}\right)$. The Dedekind $\eta$-function is $x^{1 / 24}$ times the Euler product. Let $\mathfrak{g}$ be a complex simple Lie algebra, and let $K$ be a simply connected compact group such that $\mathfrak{k}=$ Lie $K$ is a compact form of $\mathfrak{g}$. Let $\ell$ be the rank of $K$ and let $T \subset K$ be a maximal torus of $K$. Macdonald has given a formula for the $\operatorname{dim} K$ power of the Euler product in terms of a summation over a lattice in $\mathfrak{h}$. See [Ma-1]. A new understanding of this formula was made by V. Kac. It arose from his dominator identity. See Chapter 12 in [Ka]. Another approach to the formula was a consequence of the Laplacian determination (an infinite dimensional analogue of Theorem 5.14 in [Ko-3]) of the homology of the "nilradical" of the standard maximal parabolic subalgebra of the affine Kac-Moody Lie algebra. This is due to H. Garland in $[\mathrm{G}]$. Because the Laplacian is positive semidefinite this approach has the advantage of implying some very important inequalities. Garland's result is a special case of a much more general result of Shrawan Kumar. See [Ku-1] (or Theorem 3.4.2 in $[\mathrm{Ku}-2])$. Kumar's Theorem 3.5.2 is a far reaching infinite dimensional analogue of Theorem 5.7 in $[\mathrm{Ko}-1]$.

Let $\mathfrak{h}=i$ Lie $T$ and identify $\mathfrak{h}$ with its dual using the Killing form so that $\Delta \subset \mathfrak{h}$ where $\Delta$ is the set of roots for the pair $\left(\mathfrak{h}_{\mathbb{C}}, \mathfrak{g}\right)$. Let $\mathfrak{h}^{+} \subset \mathfrak{h}$ be a Weyl chamber defined by a choice, $\Delta_{+}$, of positive roots and let $D \subset \mathfrak{h}^{+}$be the set of dominant integral forms on $\mathfrak{h}$. For each $\lambda \in D$ let $\pi_{\lambda}: K \rightarrow A u t V_{\lambda}$ be an irreducible representation with highest weight $\lambda$. Let $\chi_{\lambda}$ be the character of $\pi_{\lambda}$ and let $\operatorname{Cas}(\lambda)$ be the scalar value taken by the Casimir element (relative to the Killing form) on $V_{\lambda}$. Let $\rho$ be one half

* Research supported in part by NSF contract DMS-0209473 and the KG\&G Foundation. 
the sum of the positive roots and let $a_{P}=\exp 2 \pi i 2 \rho$. An element in $K$ conjugate to $a_{P}$ is referred to in [Ko-3] as an element of type $\rho$. Using Macdonald's formula the following result was established as part of Theorem 3.1 in [Ko-3].

Theorem 0.1. For any $\lambda \in D$ one has $\chi_{\lambda}\left(a_{P}\right) \in\{-1,0,1\}$ and

$$
\left(\prod_{n=1}^{\infty}\left(1-x^{n}\right)\right)^{\operatorname{dim} K}=\sum_{\lambda \in D} \chi_{\lambda}\left(a_{P}\right) \operatorname{dim} V_{\lambda} x^{\operatorname{Cas}(\lambda)}
$$

For a heat kernel (on $K$ ) interpretation of $(0.1)$ see $\S 5$ in $[\mathrm{Ko}-3]$ or $[\mathrm{F}]$.

Of course the only dominant weights $\lambda$ which contribute to the sum (0.1) are those in the subset $\left\{\lambda \in D \mid \chi_{\lambda}\left(a_{P}\right) \in\{-1,1\}\right.$. The determination of this subset is implicit in Lemma 3.5.2 of [Ko-3]. This is clarified in $\S 2.2$ of the present paper. In more detail let $W_{f}$ be the affine Weyl group operating in $\mathfrak{h}$ and let $A_{1}=\left\{x \in \mathfrak{h}^{+} \mid \psi(x) \leq 1\right\}$ where $\psi \in \Delta_{+}$is the highest root. Then $A_{1}$ is a fundamental domain for the action of $W_{f}$ and any subset of form $A_{\sigma}=\sigma\left(A_{1}\right)$, for $\sigma \in W_{f}$, is referred to as an alcove. Let $W_{f}^{+}=\left\{\sigma \in W_{f} \mid A_{\sigma} \subset \mathfrak{h}^{+}\right\}$. An alcove $A_{\sigma}$ is called dominant if $\sigma \in W_{f}^{+}$. (A study of the set of dominant alcoves was made by Bott in [B] in connection with the topology of the loop group $\Omega(K)$ ). The set of dominant alcoves, or rather $W_{f}^{+}$, parameterize a subset $D_{\text {alcove }}$ of $D$ by defining $\lambda^{\sigma}=\sigma(2 \rho) / 2-\rho$, for any $\sigma \in W_{f}^{+}$, and putting $D_{\text {alcove }}=\left\{\lambda^{\sigma} \mid \sigma \in W_{f}^{+}\right\}$. The element $2(\rho+\lambda)$ is in the interior of $A_{\sigma}$ and in fact, if $\mathfrak{g}$ is simply-laced, this element is the center of the inscribed sphere of the simplex $A_{\sigma}$. (See the cautionary statement about $\lambda^{\sigma}$ in Remark 3.4). Let $b_{k}, k \in \mathbb{Z}_{+}$, be the coeficients defined by the expansion $\left(\prod_{n=1}^{\infty}\left(1-x^{n}\right)\right)^{\operatorname{dim} K}=\sum_{k=0}^{\infty} b_{k} x^{k}$. One then has (taken from Theorem 2.4 and (3.40))

Theorem 0.2. Let $\lambda \in D$. Then $\chi_{\lambda}\left(a_{P}\right) \in\{-1,1\}$ if and only if $\lambda \in D_{\text {alcove }}$ so that in such a case $\lambda=\lambda^{\sigma}$ for a unique $\sigma \in W_{f}^{+}$. Moreover in such a case $\chi_{\lambda^{\sigma}}\left(a_{P}\right)=(-1)^{\ell(\sigma)}$ where $\ell(\sigma)$ is the length of $\sigma$. In addition $\operatorname{Cas}\left(\lambda^{\sigma}\right) \in \mathbb{Z}_{+}$and one has the finite sum

$$
b_{k}=\sum_{\sigma \in W_{f}^{+}, \operatorname{Cas}\left(\lambda^{\sigma}\right)=k}(-1)^{\ell(\sigma)} \operatorname{dim} V_{\lambda^{\sigma}}
$$

Remark 0.3. If $K=S U(m)$ then as one knows the representation theory of $S U(m)$ defines a bijection

$$
D \rightarrow P
$$

where $P$ is the set of partitions of length at most $m-1$. For any $p \in P$ one defines another partition $\tilde{p}$ called its $m$-core. One says that $p$ has null $m$-core if $\tilde{p}$ is the empty partition. From the first statement in Theorem 0.2 it follows from Exercise 1.1.8(c) in [M] or $\S 3.4$ in $[\mathrm{A}-\mathrm{F}]$ or p. $467-469$ in [St] that the image of $D_{\text {alcove }}$ in $(0.3)$ is exactly 
the set of $p \in P$ with empty $m$-core. Such a partition exists only if the size of $p$ is an integral multiple $m k$ of $m$ and using, for example results of Bott, one can show, in this case, that the number of such partitions is $\left(\begin{array}{c}m+k-2 \\ m-2\end{array}\right)$.

0.2. Let $\hat{\mathfrak{g}}$ be the affine Kac-Moody Lie algebra corresponding to $\mathfrak{g}$. The "nil radical" of a standard maximal parabolic subalgebra is either $\mathfrak{u}^{-}=t^{-1} \mathfrak{g}\left[t^{-1}\right]$ or the opposed algebra $\mathfrak{u}=t \mathfrak{g}[t]$. The exterior algebra $\wedge \mathfrak{u}^{-}$is bigraded by $\mathbb{Z}_{+} \times \mathbb{Z}_{+}$with homogeneous components $\left(\wedge^{n} \mathfrak{u}^{-}\right)_{k}$ where $-k$ is the $t$-degree. Furthermore $\wedge \mathfrak{u}^{-}$is an underlying space for the chain complex whose derived homology is $H_{*}\left(\mathfrak{u}^{-}\right)$. In addition it is also the underlying space for a cochain complex whose derived cohomology $\mathcal{H}(\mathfrak{u})$ is a restricted form (see $\S 4.3)$ of $H^{*}(\mathfrak{u})$. Garland's theorem (Theorem 3.10 here), Theorem 3.13 and Theorem 4.8 yield

Theorem 0.4. As $\mathfrak{g}$-modules, $H_{*}\left(\mathfrak{u}^{-}\right)$and $H^{*}(\mathfrak{u})$ are equivalent and multiplicity free. In fact

$$
\begin{aligned}
H_{*}\left(\mathfrak{u}^{-}\right) & \equiv \mathcal{H}(\mathfrak{u}) \\
& \equiv \sum_{\sigma \in W_{f}^{+}} V_{\lambda^{\sigma}}
\end{aligned}
$$

With respect to the bigrading

$$
\begin{aligned}
\left(H_{n}\left(\mathfrak{u}^{-}\right)\right)_{k} & \equiv\left(\mathcal{H}^{n}(\mathfrak{u})\right)_{k} \\
& \equiv \sum_{\sigma \in W_{f}^{+}, \ell(\sigma)=n, \operatorname{Cas}\left(\lambda^{\sigma}\right)=k} V_{\lambda^{\sigma}}
\end{aligned}
$$

0.3. For $k \in \mathbb{Z}_{+}$let $C_{k} \subset \wedge^{k} \mathfrak{g}$ be the span of all 1-dimensional subspaces of the form $\wedge^{k} \mathfrak{a}$ where $\mathfrak{a} \subset \mathfrak{g}$ is any $k$-dimensional abelian subalgebra of $\mathfrak{g}$. Let $M$ be the maximal dimension of a commutative subalgebra of $\mathfrak{g}$. Obviously $C_{k} \neq 0$ if and only if $k \leq M$. The value of $M$ was computed by Malcev for each $\mathfrak{g}$-type (e.g. $M=36$ if the $\mathfrak{g}$ type is $E_{8}$ ).

If $\mathfrak{v} \subset \mathfrak{g}$ is any (complex) subspace of $\mathfrak{g}$ which is stable under $a d \mathfrak{h}$, let $\Delta(\mathfrak{v})=$ $\left\{\varphi \in \Delta \mid e_{\varphi} \in \mathfrak{v}\right\}$ where $e_{\varphi}$ is a root vector for $\varphi$. Let $\mathfrak{b} \subset \mathfrak{g}$ be the Borel subalgebra containing $\mathfrak{h}$ such that $\Delta(\mathfrak{b})=\Delta_{+}$and let $\mathfrak{n}=[\mathfrak{b}, \mathfrak{b}]$. Let $\Xi$ be an index set parameterizing the set of all abelian ideals of $\mathfrak{b}$ and for any $\xi \in \Xi$ let $\mathfrak{a}_{\xi}$ be the corresponding ideal. It is immediate that $\mathfrak{a}_{\xi} \subset \mathfrak{n}$. Let

$$
\lambda_{\xi}=\sum_{\varphi \in \Delta\left(\mathfrak{a}_{\xi}\right)} \varphi
$$

Let $\Xi_{k}=\left\{\xi \in \Xi \mid \operatorname{dim} \mathfrak{a}_{\xi}=k\right\}$. If $\xi \in \Xi_{k}$ let $V_{\xi} \subset \wedge^{k} \mathfrak{g}$ be the $\mathfrak{g}$-submodule generated by $\wedge^{k} \mathfrak{a}_{\xi}$ with respect to the adjoint action of $\mathfrak{g}$ on $\wedge \mathfrak{g}$. Obviously $V_{\xi} \subset C_{k}$. Furthermore it is immediate that $V_{\xi}$ is irreducible, $\wedge^{k} \mathfrak{a}_{\xi}$ is the highest weight space in $V_{\xi}$ and $\lambda_{\xi}$ is the highest weight of $V_{\xi}$. Moreover we have proved (see Theorem 4.2) the following result as part of Theorem (8) in [Ko-3]. 
Theorem 0.5. For any $\mathfrak{k} \in \mathbb{Z}_{+}$where $0 \leq k \leq M$ one has the direct sums

$$
C_{k}=\sum_{\xi \in \Xi_{k}} V_{\xi}
$$

and

$$
C=\sum_{\xi \in \Xi} V_{\xi}
$$

Furthermore $C$ and, a fortiori, $C_{k}$, are multiplicity-free $\mathfrak{g}$-modules.

It is a beautiful later result of Dale Peterson that $\operatorname{card} \Xi=2^{\ell}$. A simpler proof of Peterson's theorem was given in [C-P]. See Theorem 2.9 in that reference. It is clear that there are $2^{\ell}$ alcoves in $2 A_{1}$. They are parameterized by $W_{f}^{(2)}=\left\{\sigma \in W_{f}^{+} \mid\right.$ $\left.A_{\sigma} \subset 2 A_{1}\right\}$. The Cellini-Papi proof of Peterson's theorem is obtained by establishing a bijection $\Xi \rightarrow W_{f}^{(2)}$. The bijection is stated in a new way here (Theorem 0.6 below ) using $D_{\text {alcove }}$ and the weights $\left\{\lambda_{\xi} \mid \xi \in \Xi\right\}$. Recently R. Suter in [Su] has shown the Peterson's theorem follows from Theorem (8) in [Ko-3]. With the benefit of this knowledge we have found a new proof of Theorem 0.6 without the use of [C-P]. The following statement is Theorem 4.3.

Theorem 0.6. For any $\xi \in \Xi$ there exists an (necessarily unique) element $\sigma_{\xi} \in$ $W_{f}^{+}$such that

$$
\lambda_{\xi}=\lambda^{\sigma_{\xi}}
$$

Moreover $\sigma_{\xi} \in W_{f}^{(2)}$ and the map

$$
\xi \rightarrow W_{f}^{(2)}, \quad \xi \mapsto \sigma_{\xi}
$$

is a bijection. In particular one has the inclusion

$$
\left\{\lambda_{\xi} \mid \xi \in \Xi\right\} \subset D_{\text {alcove }}
$$

and the count (Peterson's theorem)

$$
\operatorname{card} \Xi=2^{\ell}
$$

Remark 0.7. Suter in $[\mathrm{Su}]$ has independently discovered (0.9). $4.5)$

The $2^{\ell}$ element subset $\left\{\Lambda^{\sigma} \mid \sigma \in W_{f}^{(2)}\right\}$ of $D_{\text {alcove }}$ is characterized in (see Theorem

Theorem 0.8. Let $\sigma \in W_{f}^{+}$. Then

$$
\operatorname{Cas}\left(\lambda^{\sigma}\right) \geq \ell(\sigma)
$$


and equality occurs in (0.13) if and only if $\sigma \in W_{f}^{(2)}$. Furthermore in that case writing $\sigma=\sigma_{\xi}$ for $\xi \in \Xi$ (Theorem 0.6) one has

$$
\begin{aligned}
\operatorname{Cas}\left(\lambda^{\sigma}\right) & =\ell(\sigma) \\
& =\operatorname{dim} \mathfrak{a}_{\xi}
\end{aligned}
$$

Cup product $(\vee)$ defines the structure of an algebra on $\mathcal{H}(\mathfrak{u})$. Theorem 0.8 has implications on the determination of an important subalgebra of $\mathcal{H}(\mathfrak{u})$. Following a suggestion of Pavel Etingof, introduce a new grading, $\mathcal{H}^{[j]}(\mathfrak{u}), j \in \mathbb{Z}_{+}$, in $\mathcal{H}(\mathfrak{u})$ by putting $\mathcal{H}^{[j]}(\mathfrak{u})=\sum_{n, k \in \mathbb{Z}, k-n=j}\left(\mathcal{H}^{n}(\mathfrak{u})\right)_{k}$. The homogeneous components are finite dimensional and one has

$$
\mathcal{H}(\mathfrak{u})=\sum_{j=0}^{\infty} \mathcal{H}^{[j]}(\mathfrak{u})
$$

and

$$
\mathcal{H}^{[j]}(\mathfrak{u}) \vee \mathcal{H}^{\left[j^{\prime}\right]}(\mathfrak{u}) \subset \mathcal{H}^{\left[j+j^{\prime}\right]}(\mathfrak{u})
$$

In particular $\mathcal{H}^{[0]}(\mathfrak{u})$ is a finite dimensional subalgebra of $\mathcal{H}(\mathfrak{u})$. See Proposition 4.12. Etingof suggested that our results should yield the structure of $\mathcal{H}^{[0]}(\mathfrak{u})$. Indeed this the case. Identify $\mathfrak{g}$ with its dual using the Killing form. Then $d: \mathfrak{g} \rightarrow \wedge^{2} \mathfrak{g}$ where $d$ is the Cartan-Eilenberg-Koszul coboundary operator whose derived cohomology is $H^{*}(\mathfrak{g})$. Let $(d \mathfrak{g})$ be the ideal in $\wedge \mathfrak{g}$ generated by $d \mathfrak{g}$. Theorem 4.3 of [Ko-6] establishes that one has the following direct sum

$$
\wedge \mathfrak{g}=C \oplus(d \mathfrak{g})
$$

so that $C$ inherits an algebra structure. I had no idea of the meaning of this algebra when [Ko-6] was written. Its meaning is clarified in (see Theorem 4.16 in the present paper)

Theorem 0.9. As a $\mathfrak{g}$-module $\mathcal{H}^{[0]}(\mathfrak{u})$ is multiplicity-free with $2^{\ell}$ irreducible components. In fact (see (0.8))

$$
\mathcal{H}^{[0]}(\mathfrak{u}) \equiv \sum_{\xi \in \Xi} V_{\xi}
$$

As an algebra (under cup product)

$$
\mathcal{H}^{[0]}(\mathfrak{u}) \equiv \wedge \mathfrak{g} /(d \mathfrak{g})
$$

Let $(d \mathfrak{g})^{k}=(d \mathfrak{g}) \cap \wedge^{k} \mathfrak{g}$. Also let $\theta($ Cas $) \in$ End $\wedge \mathfrak{g}$ be the action of Cas induced by the adjoint representation of $\mathfrak{g}$ on $\wedge \mathfrak{g}$. Then (2.1.7) and Theorem (5) in [Ko-2] yield 
Theorem 0.10. Let $k \in \mathbb{Z}_{+}$. Then the following four numbers are all equal

$$
\begin{aligned}
& \text { [1] } \operatorname{dim} C_{k} \\
& \text { [2] } \operatorname{dim} \wedge^{k} \mathfrak{g} /(d \mathfrak{g})^{k} \\
& \text { [3] } \operatorname{dim}\left\{v \in \wedge^{n} \mathfrak{g} \mid \theta(\text { Cas }) v=k v\right\} \\
& \text { [4] } \operatorname{dim}\left(\mathcal{H}^{k}(\mathfrak{u})\right)_{k}
\end{aligned}
$$

0.4. One difficulty in using (0.2) to compute the coefficient $b_{k}$ is the alternation in signs in (0.2). By Theorem 0.8 this difficulty would disappear if $k$ were such that $\operatorname{Cas}\left(\lambda^{\sigma}\right)=k$ implies that $\sigma \in W_{f}^{(2)}$. But this is the case if $k \leq h^{\vee}$ where $h^{\vee}$ is the dual Coxeter number. The following is one our main results (see Theorem 4.23).

Theorem 0.11. Let $k \leq h^{\vee}$. The following seven number are all equal.

$$
\begin{aligned}
& \text { [1] }(-1)^{k} b_{k} \\
& \text { [2] } \operatorname{dim} C_{k} \\
& \text { [3] } \sum_{\xi \in \Xi_{k}} \operatorname{dim} V_{\xi} \\
& \text { [4] } \operatorname{dim}\left\{v \in \wedge^{k} \mathfrak{g} \mid \theta(\text { Cas }) v=k v\right\} \\
& \text { [5] } \operatorname{dim} \wedge^{k} \mathfrak{g} /(d \mathfrak{g})^{k} \\
& \text { [6] } \operatorname{dim} \mathcal{H}^{k}(\mathfrak{u}) \\
& \text { [7] } \operatorname{dim} H_{k}\left(\mathfrak{u}^{-}\right)
\end{aligned}
$$

Example. If $K=S U(5)$ then since $\operatorname{dim} S U(5)=24$ one has $b_{n}=\tau(n+1)$ where $n \mapsto \tau(n)$ is the Ramanujan tau function. Here $h^{\vee}=5$. Theorem 0.11 says $\wedge$ Lie $\operatorname{Sl}(5, \mathbb{C})$ "sees" the first five non-trivial Ramanujan numbers. One has $\tau(2)=$ $-24, \tau(3)=252, \tau(4)=-1472, \tau(5)=4830 \tau(6)=-6048$. See [Se], p.97. One readily checks that (choosing [4] in Theorem 0.11 for the computation),

$$
\begin{aligned}
& \operatorname{dim} C_{1}=24 \\
& \operatorname{dim} C_{2}=252 \\
& \operatorname{dim} C_{3}=1472 \\
& \operatorname{dim} C_{4}=1472 \\
& \operatorname{dim} C_{5}=6048
\end{aligned}
$$

P. Etingof points out that Theorem 0.11 can be expressed as a homology acyclicity statement. Let $\partial_{-}$be the boundary operator on $\wedge \mathfrak{u}^{-}$whose derived homology is $H_{*}\left(\mathfrak{u}^{-}\right)$. The restriction of $\partial_{-}$to $\left(\wedge \mathfrak{u}^{-}\right)_{k}$, for any $k \in \mathbb{Z}_{+}$, defines a finite dimensional subcomplex (i.e. the $t$-degree is fixed to be $-k$ )

$$
\left(\wedge^{k} \mathfrak{u}^{-}\right)_{k} \longrightarrow\left(\wedge^{k-1} \mathfrak{u}^{-}\right)_{k} \longrightarrow \cdots \longrightarrow\left(\wedge^{0} \mathfrak{u}^{-}\right)_{k} \longrightarrow 0
$$


We thank Etingof for the following statement (see Theorem 4.24).

Theorem 0.12. If $k \leq h^{\vee}$ then the complex (0.18) is acyclic. That is, $\left(H_{n}\left(\mathfrak{u}^{-}\right)\right)_{k}=$ 0 unless $n=k$ so that $\left(H_{*}\left(\mathfrak{u}^{-}\right)\right)_{k}=\left(H_{k}\left(\mathfrak{u}^{-}\right)\right)_{k}$. In fact $\left(H_{*}\left(\mathfrak{u}^{-}\right)\right)_{k}=H_{k}\left(\mathfrak{u}^{-}\right)$and hence

$$
\operatorname{dim}\left(H_{*}\left(\mathfrak{u}^{-}\right)\right)_{k}=(-1)^{k} b_{k}
$$

0.5. One can raise the Euler product to the $s$ power where $s$ is any complex number, by taking its logarithm, multiplying by $s$ and exponentiating. One then easily has that

$$
\left(\Pi_{n=1}^{\infty}\left(1-x^{n}\right)\right)^{s}=\sum_{k=0}^{\infty} f_{k}(s) x^{k}
$$

where $f_{k}(s)$ is a polynomial of degree $k$. Obviously

$$
b_{k}=f_{k}(\operatorname{dim} K)
$$

Although one can give an expression for these polynomials the expression yields very little understanding of the nature of the polynomials. One approach could be a determination of the roots of the $f_{k}$. It is a long standing question (see p. 98 in [Se]) about the Ramanujan numbers as to whether 24 is ever a root of the $f_{k}$. The following result suggests a possible Lie-theoretic connection with the roots. Obviously $f_{1}=1$ and 0 is a root of $f_{k}$ for all $k>0$. Consider $f_{2}, f_{3}$ and $f_{4}$. Since 4 is neither a pentagonal number nor a triangular number it follows from a formula $(\mathrm{s}=1)$ of Euler and $(\mathrm{s}=3)$ of Jacobi that for one missing root $r_{4}$ one has $f_{4}(s)=1 / 4 ! s(s-1)(s-3)\left(s-r_{4}\right)$. Similarly $r_{3}$ and $r_{2}$ exist so that $-f_{3}(s)=1 / 3 ! s(s-1)\left(s-r_{3}\right)$ and $f_{2}(s)=1 / 2 ! s\left(s-r_{2}\right)$. On the other hand the only cases where $M<h^{\vee}$ are when $\mathfrak{g}$ is of type $A_{1}, A_{2}$ and $G_{2}$. As a consequence of Theorem 0.11 one has (Theorem 4.27)

Theorem 0.13. The missing roots $r_{4}, r_{3}$ and $r_{2}$ are, respectively, the complex dimensions of $G_{2}, A_{2}$ and $A_{1}$, namely 14,8 and 3 so that

$$
\begin{aligned}
f_{4}(s) & =1 / 4 ! s(s-1)(s-3)(s-14) \\
-f_{3}(s) & =1 / 3 ! s(s-1)(s-8) \\
f_{2}(s) & =1 / 2 ! s(s-3)
\end{aligned}
$$

Let $k$ be any positive integer. If $m \in \mathbb{Z}_{+}$and $m \geq 2$ let $C_{k}(m)$ equal $C_{k}$ for the case where $K=S U(m)$. If $m \geq k$ then $k \leq h^{\vee}=m$ and hence, by Theorem 0.11,

$$
f_{k}\left(m^{2}-1\right)=(-1)^{k} \operatorname{dim} C_{k}(m)
$$

The following result implies that $f_{k}(s)$ is encoded in the $k$-dimensional commutative subalgebra structure of $\operatorname{Lie} S l(m, \mathbb{C})$ for $k$ different values of $m$ where $m \geq k$ and $m>1$ (see Theorem 4.28). 
Theorem 0.14. Let $k$ be a positive integer. Then $f_{k}(s)$ is determined by the numbers $\operatorname{dim} C_{k}(m)$ for $k$ different values of $m \in \mathbb{Z}_{+}$where $m \geq k$ and $m>1$.

0.6. We wish to thank Pavel Etingof, Shrawan Kumar and Richard Stanley for valuable informative conversations.

\section{Alcoves and the affine Weyl group}

1.1. Let $\mathfrak{g}$ be a complex simple Lie algebra. The value of the Killing form $B$ of $\mathfrak{g}$ on $x, y \in \mathfrak{g}$ will be denoted by $(x, y)$ and using $B$ we will identify $\mathfrak{g}$ with its dual space. Let $\mathfrak{k}$ be a compact form of $\mathfrak{g}$ and let $K$ be a corresponding simply connected compact Lie group. Let $T$ be a maximal torus of $K$ and let $\mathfrak{t}=$ Lie $T$. Let $\mathfrak{t}_{\mathbb{C}} \subset \mathfrak{g}$ be the complexification of $\mathfrak{t}$ so that $\mathfrak{t}_{\mathbb{C}}$ is a Cartan subalgebra of $\mathfrak{g}$. The restriction of $B$ to $\mathfrak{t}_{\mathbb{C}}$ will be used to identify $\mathfrak{t}_{\mathbb{C}}$ with its dual space so that $\Delta \subset \mathfrak{t}_{\mathbb{C}}$ where $\Delta$ is set of roots for the pair $\left(\mathfrak{t}_{\mathbb{C},} \mathfrak{g}\right)$. The span of $\Delta$ over $\mathbb{R}$ is a real form $\mathfrak{h}$ of $\mathfrak{t}_{\mathbb{C}}$. In fact $\mathfrak{h}=i \mathfrak{t}$ and one knows that $B \mid \mathfrak{h}$ is positive definite.

Let $\ell=\operatorname{rank} \mathfrak{k}$ and let $I=\{1, \ldots, \ell\}$. Let $\Delta_{+} \subset \Delta$ be a choice of a positive root system and let $\Pi$ be the set of simple positive roots. The elements of $\Pi$ will be indexed by $I$ so that we can write $\Pi=\left\{\alpha_{i}\right\}, i \in I$. Let $\varepsilon$ be the epimorphism

$$
\varepsilon: \mathfrak{h} \rightarrow T
$$

where $\varepsilon(x)=\exp 2 \pi i x$. Let $\Gamma$ be the kernel of $\varepsilon$ so that $\Gamma$ is a lattice in $\mathfrak{h}$. For any $\varphi \in \Delta$ one knows that

$$
\varphi^{\vee} \in \Gamma
$$

where $\varphi^{\vee}=2 \varphi /(\varphi, \varphi)$. Furthermore the set of elements $\left\{\alpha_{i}^{\vee}\right\}, i \in I$ is a basis of $\Gamma$ so that any $\gamma \in \Gamma$ can be uniquely written

$$
\gamma=\sum_{i \in I} m_{i} 2 \alpha_{i} /\left(\alpha_{i}, \alpha_{i}\right)
$$

1.2. For any $z \in \mathfrak{h}$ let $t_{z}: \mathfrak{h} \rightarrow \mathfrak{h}$ be the translation map by $z$ so that $t_{z}(x)=z+x$ for any $x \in \mathfrak{h}$. Let $W$ be the Weyl group for the pair $(T, K)$. Obviously $\Gamma$ is stabilized by $W$ with respect to the action of $W$ on $\mathfrak{h}$. Consequently the translation group $\widetilde{\Gamma}=\left\{t_{\gamma} \mid \gamma \in \Gamma\right\}$ is normalized by $W$. The affine Weyl group $W_{f}$ is the semidirect product

$$
W_{f}=\widetilde{\Gamma} \rtimes W
$$

and we will be mainly concerned with its natural affine action on $\mathfrak{h}$.

For any $n \in \mathbb{Z}$ and $\varphi \in \Delta_{+}$let $\mathfrak{h}_{\varphi, n}$ be the hyperplane in $\mathfrak{h}$ defined by putting

$$
\mathfrak{h}_{\varphi, n}=\left\{x \in \mathfrak{h}_{\mathbb{R}} \mid(\varphi, x)=n\right\}
$$

We will use the word wall to refer to a hyperplane in $\mathfrak{h}$ of this form. More specifically a wall of the form $\mathfrak{h}_{\varphi, n}$ will referred to as a $\varphi$-wall. An element $x \in \mathfrak{h}$ will be called $W_{f}$-singular if $x \in \operatorname{Sing}(\mathfrak{h})$ where

$$
\operatorname{Sing}(\mathfrak{h})=\bigcup_{\varphi \in \Delta_{+}, n \in \mathbb{Z}} \mathfrak{h}_{\varphi, n}
$$


An element $y \in \mathfrak{h}$ will be called $W_{f}$-regular if $y$ lies in the complement $\operatorname{Reg}(\mathfrak{h})$ of $\operatorname{Sing}(\mathfrak{h})$ in $\mathfrak{h}$. The closure $A$ of a connected component of $\operatorname{Reg}(\mathfrak{h})$ is called an alcove. The connected component itself is clearly $\operatorname{Reg}(A)$ where $\operatorname{Reg}(A)=A \cap \operatorname{Reg}(\mathfrak{h})$ and one readily has that $\operatorname{Reg}(A)$ is the interior of $A$. The affine Weyl group $W_{f}$ operates simply and transitively on the set $\mathcal{A}$ of all alcoves. Let $\psi \in \Delta_{+}$be the highest root. A special alcove $A_{1}$, referred to as the fundamental alcove, can be defined by

$$
A_{1}=\left\{x \in \mathfrak{h} \mid\left(\alpha_{i}, x\right) \geq 0, i \in I, \text { and }(\psi, x) \leq 1\right\}
$$

We can then index the elements of $\mathcal{A}$ by $W_{f}$ where, if $\sigma \in W_{f}$, we put $A_{\sigma}=\sigma\left(A_{1}\right)$.

Let $\sigma \in W_{f}$ and put $T_{\sigma}=\varepsilon\left(A_{\sigma}\right)$. One knows (1) that every element in $\mathfrak{h}$ is $W_{f}$-conjugate to a unique element in $A_{1},(2) \varepsilon: W_{1} \rightarrow T_{1}$ is bijective (see (1.1)), and (3) any element in $K$ is $K$-conjugate to a unique element in $T_{1}$. Since these properties are clearly preserved by the action of $W_{f}$ one immediately has

Proposition 1.1. Let $\sigma \in W_{f}$. Then (1) every element in $\mathfrak{h}$ is $W_{f}$-conjugate to a unique element in $A_{\sigma}$, (2) $\varepsilon: A_{\sigma} \rightarrow T_{\sigma}$ is bijective (see (1.1)), and (3) any element in $K$ is $K$-conjugate to a unique element in $T_{\sigma}$.

1.3. For any $(\varphi, n) \in \Delta_{+} \times \mathbb{Z}$ let $s_{\varphi, n}$ be the reflection in $\mathfrak{h}$ defined by the wall $\mathfrak{h}_{\varphi, n}$. We write $s_{\varphi}=s_{\varphi, 0}$. Of course $s_{\varphi} \in W$. However for any $n \in \mathbb{Z}$ one readily sees that

$$
s_{\varphi, n}=t_{n \varphi \vee} s_{\varphi}
$$

so that $s_{\varphi, n} \in W_{f}$. In fact one knows that $W_{f}$ is a Coxeter group with the $\ell+1$ generators $\left\{s_{i}, s_{\psi, 1}\right\}, i \in I$, where we have written $s_{i}=s_{\alpha_{i}}$. In particular one has a length function, $\sigma \mapsto \ell(\sigma)$ on $W_{f}$. For any $\varphi \in \Delta_{+}$and $\sigma \in W_{f}$ let

$$
n_{\varphi}(\sigma)=\# \text { of } \varphi \text {-walls separating } \operatorname{Reg}\left(A_{\sigma}\right) \text { from } \operatorname{Reg}\left(A_{1}\right)
$$

It follows easily that if $\sigma \in W_{f}$ then $\ell(\sigma)$ can be given geometrically by

$$
\begin{aligned}
\ell(\sigma) & =\# \text { of walls separating } \operatorname{Reg}\left(A_{\sigma}\right) \text { from } \operatorname{Reg}\left(A_{1}\right) \\
& =\sum_{\varphi \in \Delta_{+}} n_{\varphi}(\sigma)
\end{aligned}
$$

We adopt the convention that $\mathbb{Z}_{+}$is the set of non-negative integers and $\mathbb{N}$ is the set of positive integers.

Remark 1.2. Note that for any $\sigma \in W_{f}$ and any $\varphi \in \Delta_{+}$there exists $n \in \mathbb{Z}$ such that for the open and closed unit intervals, $(n, n+1)$ and $[n, n+1]$ in $\mathbb{R}$, one has

$$
\begin{aligned}
\varphi\left(\operatorname{Reg}\left(A_{\sigma}\right)\right) & \subset(n, n+1) \\
\varphi\left(A_{\sigma}\right) & \subset[n, n+1]
\end{aligned}
$$


This is immediate since it is clearly true for $A_{\sigma}=A_{1}$. One notes also that if $n \in \mathbb{Z}_{+}$ then

$$
n=n_{\varphi}(\sigma)
$$

1.4. Let $\mathfrak{h}^{+} \subset \mathfrak{h}$ be the Weyl chamber corresponding to $\Delta_{+}$so that

$$
\mathfrak{h}^{+}=\left\{x \in \mathfrak{h} \mid(\varphi, x) \geq 0, \forall \varphi \in \Delta_{+}\right\}
$$

The interior $\operatorname{Int}\left(\mathfrak{h}^{+}\right)$can be characterized by

$$
\operatorname{Int}\left(\mathfrak{h}^{+}\right)=\left\{x \in \mathfrak{h} \mid\left(\alpha_{i}, x\right)>0, \forall i \in I\right\}
$$

Let $\mathcal{A}^{+}$be set of all alcoves $A$ such that $A \subset \mathfrak{h}^{+}$. This defines a subset $W_{f}^{+}$of the affine Weyl group by the condition $\mathcal{A}^{+}=\left\{A_{\sigma} \mid \sigma \in W_{f}^{+}\right\}$. Note that by Remark 1.2 and (1.9) one has $\sigma \in W_{f}^{+}$if and only if $\operatorname{Int}\left(\mathfrak{h}^{+}\right) \cap A_{\sigma} \neq \emptyset$. It follows easily then that

$$
\mathfrak{h}^{+}=\bigcup_{\sigma \in W_{f}^{+}} A_{\sigma}
$$

Remark 1.3. One readily shows that $W_{f}^{+}$is the set of minimal length representatives of the right cosets of $W$ in $W_{f}$. In fact if $w \in W$ and $\sigma \in W_{f}^{+}$then

$$
\ell(w \sigma)=\ell(w)+\ell(\sigma)
$$

Indeed $\ell(\sigma)$ walls of the form $\mathfrak{h}_{\varphi, n}$, where $n \neq 0$, clearly separate $\operatorname{Reg}\left(w\left(A_{\sigma}\right)\right)$ from $\operatorname{Reg}\left(w\left(A_{1}\right)\right)$ but $\ell(w)$ walls of the form $\mathfrak{h}_{\varphi, 0}$ separate $\operatorname{Reg}\left(w\left(A_{1}\right)\right)$ from $\operatorname{Reg}\left(A_{1}\right)$.

Recall that $\psi$ is the highest root. For any integer $k \in \mathbb{Z}_{+}$let $\mathfrak{h}^{(k)}=\left\{x \in \mathfrak{h}^{+} \mid\right.$ $(\psi, x) \leq k\}$. Clearly $\mathfrak{h}^{(k)}=k A_{1}$ so that, if $k \in \mathbb{N}$, the interior of $\mathfrak{h}^{(k)}$ is given by

$$
\operatorname{Int}\left(\mathfrak{h}^{(k)}\right)=\left\{x \in \mathfrak{h} \mid\left(\alpha_{i}, x\right)>0, \forall i \in I \text { and }(\psi, x)<k\right\}
$$

Obviously $\mathfrak{h}^{(k)}$ is the closure of its interior. Furthermore since every point in $\mathfrak{h}$ lies in at most a finite number of alcoves (e.g. from volume considerations) it follows from Remark 1.2 where $\varphi=\psi$ and $\varphi=\alpha_{i}, i \in I$, that, if $k \in \mathbb{N}, \mathfrak{h}^{(k)}$ is a union of alcoves. The following very simple and neat observation (and proof) was made in [Cellini and Papi].

Proposition 1.4. There are exactly $k^{\ell}$ alcoves in $\mathfrak{h}^{(k)}$.

Proof (Cellini and Papi). Since $\mathfrak{h}^{(k)}=k A_{1}$ the volume of $\mathfrak{h}^{(k)}$ is $k^{\ell}$ times the volume of $A_{1}$. The result then follows since every alcove necessarily has the same volume. QED 
Remark 1.5. Assume $\sigma \in W_{f}^{+}$. If $\ell(\sigma)<k$ note that

$$
A_{\sigma} \subset \mathfrak{h}^{(k)}
$$

Indeed otherwise the $\psi$-walls $\mathfrak{h}_{\psi, j}, j=1, \ldots, k$, would separate $\operatorname{Reg}\left(A_{\sigma}\right)$ from $\operatorname{Reg}\left(A_{1}\right)$ contradicting the fact that $\ell(\sigma)<k$. QED

It follows from Proposition 1.4 and Remark 1.5 that there exists a formal power series $P(t)$ with coefficients in $\mathbb{Z}_{+}$such that

$$
P(t)=\sum_{\sigma \in W_{f}^{+}} t^{\ell(\sigma)}
$$

The alcoves in $\mathfrak{h}^{+}$have a well-known connection with the loop group $\Omega(K)$. See [B] and p.444 in [Ku-2]. Conforming to much of current terminology we take the exponents $\left\{m_{i}\right\}, i \in I$, of $K$ to be the positive integers (in non-decreasing order) such that the product of $\left(1+t^{2 m_{i}+1}\right)$ over $i \in I$ is the Poincaré polynomial of $K$. This makes $m_{i}$ here have value 1 less than the value assigned to $m_{i}$ in $[\mathrm{B}]$. The following is a classic result of Bott on the Poincaré series of $\Omega(K)$. See Theorem B and (13.2) in [B].

Theorem 1.6 (Bott). The Poincaré series of the loop group $\Omega(K)$ is $P\left(t^{2}\right)$. Furthermore

$$
P(t)=\prod_{i \in I} 1 /\left(1-t^{m_{i}}\right)
$$

1.5. Let $D \subset \mathfrak{h}^{+}$be the set of all dominant integral linear forms on $\mathfrak{h}$ and for each $\lambda \in D$ let $\pi_{\lambda}: K \rightarrow$ Aut $V_{\lambda}$ be an irreducible representation with highest weight $\lambda$. As usual $\pi_{\lambda}$ will also denote the corresponding representation of $\mathfrak{g}$ and the universal enveloping algebra $U(\mathfrak{g})$ on $V_{\lambda}$. Let Cas $\in \operatorname{Cent}(U(\mathfrak{g}))$ be the quadratic Casimir element corresponding to the Killing form. For any $\lambda \in D$ let $\operatorname{Cas}(\lambda)$ be the value of the infinitesmal character of $\pi_{\lambda}$ on Cas. We recall that $\operatorname{Cas}(\lambda)=(\lambda+\rho, \lambda+\rho)-(\rho, \rho)$ or

$$
\operatorname{Cas}(\lambda)=(\lambda, \lambda)+(2 \rho, \lambda)
$$

where as usual $\rho=1 / 2 \sum_{\varphi \in \Delta_{+}} \varphi$.

Recall that $\psi$ is the highest root. As in $\S 2.2$ in [Ko-3] let

$$
h_{P}=1 /(\psi, \psi)
$$

Of course $\psi$ is the highest weight of the adjoint representation so that $\operatorname{Cas}(\psi)=1$. Thus as already noted in (2.2.3) in [Ko-3] one has (see (1.16)) $1=(2 \rho, \psi)+(\psi, \psi)$. This immediately implies that

$$
h_{P}=(2 \rho, \psi) /(\psi, \psi)+1
$$


(see (2.2.4) in [Ko-3]). Since $\psi$ is a long root one has $h_{P}=1 /(\varphi, \varphi)$ for any long root and $h_{P}$ is a positive integer. Let $h$ be the Coxeter number of $\mathfrak{g}$.

In the later publication, [Ka], the number $h_{P}$ was referred as to as the dual Coxeter number and was denoted by $h^{\vee}$. It plays a major role in Kac-Moody theory. Conforming to this now well accepted terminology one has

Proposition 1.8. One has $1 /(\varphi, \varphi)$ is the dual Coxeter number $h^{\vee}$ for any long root $\varphi \in \Delta$. Furthermore $h^{\vee}=h$ if $\mathfrak{g}$ is simply-laced.

Proof. For the first statement see the argument at the end of exercise 6.2 in $\S 6.8$ of [Ka]. The last statement is Proposition 2.2 in [Ko-3]. QED

Remark 1.9. One has $1 /(\varphi, \varphi)$ is a positive integral multiple of the dual Coxeter number for any $\varphi \in \Delta$. See Proposition 2.3.1 and its proof in [Ko-3]. In particular $1 /(\varphi, \varphi)$ is a positive integer for any $\varphi \in \Delta$.

1.6. By definition (see §1.1) for any $\sigma \in W_{f}$ there uniquely exists $w^{\sigma} \in W$ and $z^{\sigma} \in \Gamma$ such that for any $x \in \mathfrak{h}$ one has

$$
\sigma(x)=w^{\sigma}(x)+z^{\sigma}
$$

Let $\Delta_{-}=-\Delta_{+}$and for any $w \in W$ let $\Phi_{w}=w\left(\Delta_{-}\right) \cap \Delta_{+}$so that, as one knows,

$$
\ell(w)=\operatorname{card} \Phi_{w}
$$

Proposition 1.10. Let $\sigma \in W_{f}^{+}$. Then

$$
\ell(\sigma)+\ell\left(w^{\sigma}\right)=\left(2 \rho, z^{\sigma}\right)
$$

Proof. Let $x \in \operatorname{Reg}\left(A_{1}\right)$ so that $y \in \operatorname{Reg}\left(A_{\sigma}\right)$ where $y=\sigma(x)$. Let $\varphi \in \Delta_{+}$. By (1.7) and (1.8) one has that

$$
n_{\varphi}(\sigma)+1>\varphi(y)>n_{\varphi}(\sigma)
$$

For notational convenience put $w=w^{\sigma}$. But since $\varphi(w(x))=w^{-1}(\varphi)(x)$ one has, by (1.19),

$$
\varphi(y)=w^{-1}(\varphi)(x)+\varphi\left(z^{\sigma}\right)
$$

But $0<\left|w^{-1}(\varphi)(x)\right|<1$. Since $\varphi\left(z^{\sigma}\right)$ is an integer, one has, by $(1.22), \varphi\left(z^{\sigma}\right)=n_{\varphi}(\sigma)$ or $n_{\varphi}(\sigma)+1$, according as $w^{-1}(\varphi) \in \Delta_{+}$or $w^{-1}(\varphi) \in \Delta_{-}$, that is, according as $\varphi \notin \Phi_{w}$ or $\varphi \in \Phi_{w}$. But then summing $\varphi\left(z^{\sigma}\right)$ over all $\varphi \in \Delta_{+}$yields (1.21), by (1.6) and (1.20). QED 


\section{Powers of the Euler product and the set of weights $D_{\text {alcove }}$}

2.1. Let $i \in \mathcal{I}$. One knows $\left(\rho, \alpha_{i}^{\vee}\right)=1$ so that

$$
\left(2 \rho, \alpha_{i}\right)=\left(\alpha_{i}, \alpha_{i}\right)
$$

On the other hand by (1.22) and (1.23) one has

$$
(2 \rho, \psi)=1-(\psi, \psi)
$$

It follows from (2.1) and (2.2) that

$$
2 \rho \in \operatorname{Reg}\left(A_{1}\right)
$$

As in [Ko-3] (see §3.1) let $a_{P} \in K$ be defined by putting $a_{P}=\exp 2 \pi i 2 \rho$. Let $a \in K$. In [Ko-3], §3.1, we said that $a$ will be called an element of type $\rho$ if it is conjugate to $a_{P}$. (Because of the factor 2 this choice of terminology is perhaps inappropriate but it will be retained nontheless.) For any $\lambda \in \Lambda$ let $\chi_{\lambda}$ be the $K$-character of the irreducible $\pi_{\lambda}$. In [Ko-3], $\S 3.1$, we proved the following theorem about the $\operatorname{dim} K$ power of the Euler product $\prod_{n=1}^{\infty}\left(1-x^{n}\right)$.

Theorem 2.1. For any $\lambda \in D$ one has $\chi_{\lambda}\left(a_{P}\right) \in\{-1,0,1\}$ and as formal power series

$$
\left(\prod_{n=1}^{\infty}\left(1-x^{n}\right)\right)^{\operatorname{dim} K}=\sum_{\lambda \in D} \chi_{\lambda}\left(a_{P}\right) \operatorname{dim} V_{\lambda} x^{\operatorname{Cas}(\lambda)}
$$

See Theorem 3.1 in $[\mathrm{Ko}-3]$.

Remark 2.2. For a relation between (2.4) and the Laplace -Beltrami operator on $K$ (and implicitly the heat kernel on $K$ ) see $\S 5$ in [Ko-3] and [F]. See also [Ze] for a recent physical application of the heat kernel aspect of (2.4).

2.2. For any $\sigma \in W_{f}^{+}$let $\lambda^{\sigma} \in \mathfrak{h}$ be defined by putting

$$
\lambda^{\sigma}=\left(w^{\sigma}(\rho)-\rho\right)+z^{\sigma} / 2
$$

Proposition 2.3. Let $\sigma \in W_{f}^{+}$. Then $\lambda^{\sigma} \in D$. Furthermore $\lambda^{\sigma}$ is in the root lattice.

Proof. Since $w^{\sigma}(\rho)$ and $\rho$ are two weights of the representation $\pi_{\rho}$ it follows that $w^{\sigma}(\rho)-\rho$ is in the root lattice. But, by (1.3), $z^{\sigma} / 2$ is in the lattice spanned by $\alpha_{i} /\left(\alpha_{i}, \alpha_{i}\right), i \in I$. Hence $z^{\sigma} / 2$ is in the root lattice by Remark 1.9. Thus $\lambda^{\sigma}$ is in the 
root lattice. In particular $\lambda^{\sigma}$ is in the weight lattice. But then $\lambda^{\sigma}+\rho$ is in the weight lattice. But by the definition (2.5) one immediately has

$$
2\left(\lambda^{\sigma}+\rho\right)=\sigma(2 \rho)
$$

Thus

$$
2\left(\lambda^{\sigma}+\rho\right) \in \operatorname{Reg}\left(A_{\sigma}\right)
$$

In particular $2\left(\lambda^{\sigma}+\rho\right) \in \operatorname{Int}\left(\mathfrak{h}^{+}\right)$. But then $\lambda^{\sigma}+\rho \in \operatorname{Int}\left(\mathfrak{h}^{+}\right)$. But this implies that $\lambda^{\sigma}$ is dominant so that $\lambda^{\sigma} \in D$. QED

Let $D_{\text {alcove }}=\left\{\lambda^{\sigma} \mid \sigma \in W_{f}^{+}\right\}$. By drawing attention to $D_{\text {alcove }}$ we have in effect isolated what will be seen to be a distinguished subset of $D$ or, more significantly, a distinguished subset of the set of all finite dimensional irreducible representations of $K$. It is obvious from (2.7) that the map

$$
W_{f}^{+} \rightarrow D_{\text {alcove }} \quad \sigma \mapsto \lambda^{\sigma}
$$

is bijective so that the subset $D_{\text {alcove }}$ is parameterized by the set of all alcoves in a Weyl chamber.

Obviously in the formula (2.4) the only contributions to the sum on the right hand side correspond to those $\lambda \in D$ such that $\chi_{\lambda}\left(a_{P}\right) \in\{-1,1\}$. We now find that this condition characterizes $D_{\text {alcove }}$.

Theorem 2.4. Let $\lambda \in D$ so that $\chi_{\lambda}\left(a_{P}\right) \in\{-1,0,1\}$. Then $\chi_{\lambda}\left(a_{P}\right) \in\{-1,1\}$ if and only if $\lambda \in D_{\text {alcove. In particular the equality (2.4) simplifies to }}$

$$
\left(\prod_{n=1}^{\infty}\left(1-x^{n}\right)\right)^{\operatorname{dim} K}=\sum_{\sigma \in W_{f}^{+}} \chi_{\lambda^{\sigma}}\left(a_{P}\right) \operatorname{dim} V_{\lambda^{\sigma}} x^{\operatorname{Cas}\left(\lambda^{\sigma}\right)}
$$

Furthermore if $\sigma \in W_{f}^{+}$then

$$
\begin{aligned}
\chi_{\lambda^{\sigma}}\left(a_{P}\right) & =(-1)^{\ell(\sigma)} \\
& =(-1)^{\ell\left(w^{\sigma}\right)}
\end{aligned}
$$

Proof. We will use results and notations of [Ko-3]. By Lemma 3.6 in loc. cit. one has

$$
\chi_{\lambda}\left(a_{P}\right)=\epsilon_{P}(\lambda)
$$

where $\epsilon_{P}(\lambda)$ is defined following Lemma 3.5.2, p. 199. The definition of $\epsilon_{P}(\lambda)$ rests upon an earlier definition of the lattice $M_{P}$. This lattice is defined in the line preceding Proposition 2.3.1. In the notation of the present paper $M_{P}=\Gamma / 2$. Lemma 3.5.2 can then be restated as follows: either (1) $v(\lambda+\rho)-\rho \notin \Gamma / 2$ for all $v \in W$ or (2) there 
exists a unique $v \in W$ such that $v(\lambda+\rho)-\rho \in \Gamma / 2$. Lemma 3.5.2 can then be restated as follows: either (1) $v(\lambda+\rho)-\rho \notin \Gamma / 2$ for all $v \in W$ or (2) there exists a unique $v \in W$ such that $v(\lambda+\rho)-\rho \in \Gamma / 2$. By definition $\epsilon_{P}(\lambda)=0$ in case (1) and $\epsilon_{P}(\lambda)=(-1)^{\ell(v)}$ in case (2). But then, by (2.11), $\chi_{\lambda}\left(a_{P}\right)=0$ in case (1) and

$$
\chi_{\lambda}\left(a_{P}\right)=(-1)^{\ell(v)}
$$

in case (2). We will prove that $\lambda \in D_{\text {alcove }}$ if and only if $\lambda$ satisfies the condition of case (2). Assume case (2). Let $v \in W$ be such that $v(\lambda+\rho)-\rho \in \Gamma / 2$. Then there exists $\gamma \in \Gamma$ such that

$$
v(2(\lambda+\rho))-2 \rho=\gamma
$$

Applying $v^{-1}$ to this equality yields

$$
2(\lambda+\rho)=w(2 \rho)+z
$$

where we have put $w=v^{-1}$ and $z=w(\gamma)$. But clearly $z \in \Gamma$ since $\Gamma$ is stable under the action of $W$. Let $\sigma \in W_{f}$ be defined by putting $\sigma=t_{z} w$. Then (2.13) asserts that

$$
2(\lambda+\rho)=\sigma(2 \rho)
$$

But $2(\lambda+\rho) \in \operatorname{Int}\left(\mathfrak{h}^{+}\right)$since $\lambda \in D$. Thus $\sigma \in W_{f}^{+}$and hence $\lambda=\lambda^{\sigma} \in D_{\text {alcove }}$ by (2.6). Also $z=z^{\sigma}$ and $w=w^{\sigma}$. But now $\ell(v)=\ell\left(w^{\sigma}\right)$ since $w^{\sigma}=v^{-1}$. But now clearly $\left(2 \rho, 2 \varphi /(\varphi, \varphi) \in 2 \mathbb{Z}\right.$ for any $\varphi \in \Delta$. Thus $\left(2 \rho, z^{\sigma}\right) \in 2 \mathbb{Z}$. But then, recalling (1.21), this implies that the parity of $\ell(\sigma)$ is the same as the parity of $\ell\left(w^{\sigma}\right)$. Consequently

$$
\chi_{\lambda^{\sigma}}\left(a_{P}\right)=(-1)^{\ell(\sigma)}
$$

by $(2.12)$.

Now conversely assume $\lambda \in D_{\text {alcove }}$ so that $\lambda=\lambda^{\sigma}$ for a unique $\sigma \in W_{f}^{+}$. Thus $2\left(\lambda^{\sigma}+\rho\right)=w^{\sigma}(2 \rho)+z^{\sigma}$ by (2.6). Applying $v$ where $v^{-1}=w^{\sigma}$ and dividing by 2 yields the relation $v(\lambda+\rho)=\rho+\gamma$ where $\gamma=v\left(z^{\sigma}\right) / 2$. But then $\gamma \in \Gamma / 2$ and hence $\lambda$ satisfies the condition of case (2). The result then follows from (2.12) and (2.14). QED

2.3. In this section we will consider the case where $K=S U(m)$ (i.e $\mathfrak{g}_{\mathbb{C}}$ is of type $A_{m-1}$. In this case combinatorists have come upon the set $D_{\text {alcove }}$ from an entirely different perspective. Let $Q$ be the set of all partitions $q=\left(q_{1}, \ldots, q_{m}\right)$ of length at most $m-1$ so that $q_{m}=0$. One has a bijection

$$
f: D \rightarrow Q
$$

where if $q=f(\lambda)$ then $\left(\lambda, \alpha_{i}^{\vee}\right)=q_{i}-q_{i+1}, i \in I$, where $e_{\alpha_{i}}$ is the matrix unit $e_{i, i+1}$.

Associated to $q \in Q$ is another partition $\widetilde{q}$ called $m$-core of $q$. See Exercise 1.1.8(c) in [Ma-2] or $\S 3.4$ in [A-F] or p. 467-469 in [St]. The partition $\widetilde{q}$ is derived from $q$ by a step-by-step process of appropriately removing, from the Young diagram of $q$, what are 
called $m$-border strips in [Ma-2], [St] or rim hooks of length $m$ in [A-F]. The process is terminated when no more removals are possible. What remains is the Young diagram of $\widetilde{q}$. The proof that $\widetilde{q}$ is uniquely determined is particularly nice in $[\mathrm{A}-\mathrm{F}]$. One says $q$ has a null $m$ core if $\widetilde{q}$ is the empty partition. Let $Q_{o}=\{q \in Q \mid q$ has null $m$ core $\}$. It is obvious that the size of any $q \in Q$ is a multiple of $m$. See Lemma 3.4 in [A-F] for a neat characterization of the elements in $Q_{o}$.

Theorem 2.5. One has $f\left(D_{\text {alcove }}\right) \subset Q_{o}$ and

$$
f: D_{\text {alcove }} \rightarrow Q_{o}
$$

is a bijection.

Proof. Let $\lambda \in D$. Then by $1.3 .17(\mathrm{a})$, p. 50 in $[\mathrm{M}]$ one has $\chi_{\lambda}\left(a_{P}\right) \in\{0,1,-1\}$ and $\chi_{\lambda}\left(a_{P}\right) \in\{1,-1\}$ if and only if $\lambda \in Q_{o}$. A similar statement is made in Theorem 5.7 of $[\mathrm{A}-\mathrm{D}]$. (Of course these are statements for the $S U(m)$ case.) The result then follows from Theorem 2.4 in the present paper. QED

Remark 2.6. Using Bott's formula (1.13), one can show that the number $q \in Q_{o}$ having size $m k$ is $\left(\begin{array}{c}m+k-2 \\ m-2\end{array}\right)$. However both R. Stanley and R. Adin have pointed out that this statement can be deduced from known facts about $Q_{o}$.

\section{The structure of the homology $H_{*}\left(\mathfrak{u}^{-}\right)$.}

3.1. The main results of this paper are given in $\S 4$. We have been convinced by Pavel Etingof that the results are best illuminated using results of Garland [G], Garland-Lepowsky [G-L] and [Ku-1,2] on the homology and cohomology of the "nilradical" of the standard maximal parabolic subalgebra of the affine Kac-Moody Lie algebra associated to $\mathfrak{g}$. For the definition of the affine Kac-Moody Lie algebra see $\S 6$ in $[\mathrm{Ka}]$ or $\S 13.1$ in $[\mathrm{Ku}-2]$. In the main we will adhere to the development of affine Kac-Moody Lie algebras in these references except for some changes in notation. In particular we retain our previous meaning of $\mathfrak{g}$ (and not write $\mathfrak{g}$ for the finite dimensional complex simple Lie algebra). Also we write $\hat{\mathfrak{g}}$ for the affine Kac-Moody Lie algebra $\hat{\mathcal{L}}(\mathfrak{g})$ given in (1), p. 482 in $[\mathrm{Ku}-2]$. One has $\mathfrak{g} \subset \hat{\mathfrak{g}}$ and if $c, d \in \hat{\mathfrak{g}}$ are as in $\S 13.1 .1$ in $[\mathrm{Ku}-2]$ then, writing $\delta$ for $c$, the complexification $\hat{\mathfrak{h}}_{\mathbb{C}}$ of the real $\ell+2$-dimensional abelian Lie subalgebra

$$
\hat{\mathfrak{h}}=\mathfrak{h}+\mathbb{R} \delta+\mathbb{R} d
$$

"serves" as a Cartan subalgebra of $\hat{\mathfrak{g}}$. Let $\mathfrak{d}=\mathbb{R} \delta+\mathbb{R} d$. We extend the positive definite bilinear form $B \mid \mathfrak{h}$ to a nonsingular bilinear form $(x, y)$ on $\hat{\mathfrak{h}}$ so that $\mathfrak{h}$ is orthogonal to $\mathfrak{d},(d, d)=(\delta, \delta)=0$ and $(d, \delta)=1$. This is further extended to $\hat{\mathfrak{h}}_{\mathbb{C}}$ by complex linearity. Using the latter extension we identify $\hat{\mathfrak{h}}_{\mathbb{C}}$ with its dual space. As a linear space one has the direct sum decomposition

$$
\hat{\mathfrak{g}}=\mathfrak{g}_{\mathfrak{o}}+t \mathfrak{g}[t]+t^{-1} \mathfrak{g}\left[t^{-1}\right]
$$


where $\mathfrak{d}_{\mathbb{C}}$ is the complexification of $\mathfrak{d}$ and we have put $\mathfrak{g}_{\mathfrak{d}}=\mathfrak{g}+\mathfrak{d}_{\mathbb{C}}$. For a parameter $u$ the space $\mathfrak{g}[u]$ is the direct sum

$$
\mathfrak{g}[u]=\sum_{k=0}^{\infty} u^{k} \mathfrak{g}
$$

The commutation relations in $\hat{\mathfrak{g}}$ are given in (2), $\$ 13.1 .1$ in [Ku-2]. In particular $\mathfrak{d}_{\mathbb{C}}=$ Cent $\mathfrak{g}_{\mathfrak{d}}$ and the adjoint action of $\mathfrak{g}$ on $\hat{\mathfrak{g}}$ stabilizes $\mathbb{C}\left[t, t^{-1}\right] \mathfrak{g}$ and is linear with respect to the $\mathbb{C}\left[t, t^{-1}\right]$-module structure on $\mathbb{C}\left[t, t^{-1}\right] \mathfrak{g}$. It is otherwise obvious. One has $a d \delta=0$ and $a d d$ is the $t$-degree operator $t \frac{d}{d t}$.

For any $\varphi \in \Delta$ let $0 \neq e_{\varphi} \in \mathfrak{g}$ be a corresponding weight vector. The set $\hat{\Delta}^{\prime}$ of affine roots is just the set of nonzero weights for the adjoint action of $\hat{\mathfrak{h}}_{\mathbb{C}}$ on $\hat{\mathfrak{g}}$. For any affine root $\beta$ let $\hat{\mathfrak{g}}_{\beta} \subset \hat{\mathfrak{g}}$ be the corresponding root space. One has the disjoint union

$$
\hat{\Delta}^{\prime}=\hat{\Delta}^{I m} \cup \hat{\Delta}
$$

where $\hat{\Delta}^{I m}=(\mathbb{Z}-\{0\}) \delta$. The elements in $\hat{\Delta}^{I m}$ are called imaginary affine roots. If $\beta$ is an imaginary affine root so that $\beta=m \delta$ for a nonzero integer $m$, then $\hat{\mathfrak{g}}_{\beta}=t^{m} \mathfrak{h}_{\mathbb{C}}$. One has

$$
\hat{\Delta}=\Delta+\mathbb{Z} \delta
$$

One notes that

$$
\hat{\Delta}^{\prime} \subset \hat{\mathfrak{h}}
$$

The elements of $\hat{\Delta}$ are called real affine roots. If $\beta$ is a real affine root, so that $\beta=\varphi+k \delta$, where $\varphi \in \Delta$ and $k \in \mathbb{Z}$, put

$$
e_{\beta}=t^{k} e_{\varphi}
$$

(this is clearly unambiguous even if $\beta=\varphi$ ) and one has

$$
\hat{\mathfrak{g}}_{\beta}=\mathbb{C} e_{\beta}
$$

One introduces the set $\hat{\Delta}_{+}^{\prime}$ of positive affine roots by putting

$$
\hat{\Delta}_{+}^{\prime}=\hat{\Delta}_{+}^{I m} \cup \hat{\Delta}_{+}
$$

where $\hat{\Delta}_{+}^{I m}=\mathbb{N} \delta$ and

$$
\hat{\Delta}_{+}=\left(\Delta_{+}+\mathbb{Z}_{+} \delta\right) \cup\left(\Delta_{-}+\mathbb{N} \delta\right)
$$

The sets obtained by multiplying each of the 3 sets in (3.8) by -1 is denoted by replacing the subscript + by the subscript - . If $\beta$ is a real affine root then clearly $(\beta, \beta)>0$ (see (3.4) and (3.5)) so that $\hat{\mathfrak{h}}_{\beta}$, defined as the orthocomplement of $\beta$ in $\hat{\mathfrak{h}}$, is a subspace of codimension 1 in $\hat{\mathfrak{h}}_{\beta}$. Let $s_{\beta}$ be the (linear) orthogonal reflection of $\hat{\mathfrak{h}}$ defined by the subspace $\hat{\mathfrak{h}}_{\beta}$. Let $I_{*}=I \cup\{0\}$ and let $\alpha_{0} \in \hat{\Delta}_{+}$be defined by putting $\alpha_{0}=\delta-\psi$. The "Weyl group" of $\hat{\mathfrak{g}}$ is the Coxeter group $\hat{W}$ with simple generators 
$\left\{s_{\alpha_{i}}\right\}, i \in I_{*}$, operating linearly on $\hat{\mathfrak{h}}$. See $\S 1.3 .1$ in $[\mathrm{Ku}-2]$. As such one has a length function $\tau \mapsto \ell(\tau)$ on $\hat{W}$. Also $s_{\beta} \in \hat{W}$ for any real affine $\operatorname{root} \beta$. Since the simple generators of $\hat{W}$ include the simple generators of $W$ one has a natural embedding of $W$ in $\hat{W}$. The definition of $\Phi_{w}$ for $w \in W$ (see $\S 1.6$ ) extends to any $\tau \in \hat{W}$ by putting $\Phi_{\tau}=\hat{\Delta}_{+}^{\prime} \cap \tau\left(\hat{\Delta}_{-}^{\prime}\right)$. Since $\delta$ is fixed under the action of $\hat{W}$ one notes that

$$
\Phi_{\tau}=\hat{\Delta}_{+} \cap \tau\left(\hat{\Delta}_{-}\right)
$$

Furthermore not only is $\Phi_{\tau}$ a finite set but in fact

$$
\ell(\tau)=\operatorname{card} \Phi_{\tau}
$$

See e.g. Lemma 1.3.14 in [Ku-2].

Let $\hat{\mathfrak{h}}_{1}$ be the hyperplane in $\hat{\mathfrak{h}}$ defined by putting $\hat{\mathfrak{h}}_{1}=\{x \in \hat{\mathfrak{h}} \mid(\delta, x)=1\}$ so that $\hat{\mathfrak{h}}_{1}=d+\mathbb{R} \delta+\mathfrak{h}$. It is clear that $\hat{\mathfrak{h}}_{1}$ is stable under the action of $\hat{W}$. Let $\zeta: \hat{\mathfrak{h}} \rightarrow \mathfrak{h}$ be the projection with kernel $\mathfrak{d}$. Then

$$
\hat{W} \rightarrow W_{f}, \quad \tau \mapsto \bar{\tau}
$$

is a group isomorphism where for any $x \in \mathfrak{h}$,

$$
\bar{\tau}(x)=\zeta(\tau(d+x))
$$

See $\S 6.6$ in $[\mathrm{Ka}]$. If $\varphi \in \Delta_{+}, n \in \mathbb{N}$ and and $k \in \mathbb{Z}_{+}$one readily notes that

$$
\begin{aligned}
& \overline{s_{n \delta-\varphi}}=s_{\varphi, n} \\
& \overline{s_{k \delta+\varphi}}=s_{\varphi,-k}
\end{aligned}
$$

See e.g. p. 132 in $[\mathrm{C}-\mathrm{P}]$. Extend the map (3.12) to be an involutory bijection on the set $\hat{W} \cup W$ by putting

$$
\bar{\sigma}=\tau
$$

where $\sigma \in W_{f}$ and $\tau \in \hat{W}$ is such that $\bar{\tau}=\sigma$.

Let $\sigma \in W_{f}^{+}$. It is clear that any wall which separates $\operatorname{Reg}\left(A_{1}\right)$ from $\operatorname{Reg}\left(A_{\sigma}\right)$ is necessarily of the form $\mathfrak{h}_{\varphi, n}$ where $n>0$ and $\varphi \in \Delta_{+}$. On the other hand it is immediate, say, from Lemma 1.3.14 in [Ku-2] and (3.14), (See also (1.1), p. 132 in $[\mathrm{C}-\mathrm{P}])$ that $\mathfrak{h}_{\varphi, n}$ is such a wall if and only if $n \delta-\varphi \in \Phi_{\bar{\sigma}}$. That is, one has

Proposition 3.1. Let $\sigma \in W_{f}^{+}$. Then

$$
\Phi_{\bar{\sigma}}=\left\{n \delta-\varphi \mid \mathfrak{h}_{\varphi, n} \text { separates } \operatorname{Reg}\left(A_{1}\right) \text { from } \operatorname{Reg}\left(A_{\sigma}\right)\right\}
$$

3.2. Let

$$
\hat{\rho}=d / 2+\rho
$$


We note that for $i \in I_{*}$ one has

$$
\left(\hat{\rho}, \alpha_{i}\right)=\left(\alpha_{i}, \alpha_{i}\right) / 2
$$

Indeed the equality (3.17) is well known for $i \in I$. Since $\alpha_{0}=\delta-\psi$, for $i=0$, one has

$$
\begin{aligned}
\left(\hat{\rho}, \alpha_{0}\right) & =1 / 2-(\rho, \psi) \\
& =(\psi, \psi) / 2 \\
& =\left(\alpha_{0}, \alpha_{0}\right)
\end{aligned}
$$

by (1.17) and (1.18). This proves (3.17).

For any subset $\Phi \subset \hat{\Delta}_{+}$let $\langle\Phi\rangle=\sum_{\beta \in \Phi} \beta$. For the proof of the following ((3.18)) extension of (5.10.1) in [Ko-1] to the Kac-Moody case see Proposition 2.5 in [G-L] or (3) in Corollary 1.3.22 in [Ku-2]. For any $\tau \in \hat{W}$ one has

$$
\hat{\rho}-\tau(\hat{\rho})=\left\langle\Phi_{\tau}\right\rangle
$$

For any $\sigma \in W_{f}$ and $\varphi \in \Delta_{+}$recall the definition, in (1.5), of the integer $n_{\varphi}(\sigma)$.

Lemma 3.2. Let $\sigma \in W_{f}^{+}$. Then

$$
\hat{\rho}-\bar{\sigma}(\hat{\rho})=\left(\sum_{\varphi \in \Delta_{+}} n_{\varphi}(\sigma)\left(n_{\varphi}(\sigma)+1\right) / 2\right) \delta-\left(\sum_{\varphi \in \Delta_{+}} n_{\varphi}(\sigma) \varphi\right)
$$

Proof. Let $\varphi \in \Delta_{+}$. It is immediate from (1.7) and (1.8) that the wall $\mathfrak{h}_{\varphi, j}$ separates $\operatorname{Reg}\left(A_{1}\right)$ from $\operatorname{Reg}\left(A_{\sigma}\right)$ if and only if $j$ is a positve integer such that $1 \leq j \leq$ $n_{\varphi}(\sigma)$. But then (3.19) follows immediately from Proposition 3.1 and (3.18) where $\tau=\bar{\sigma}$. QED

One now has the following explicit expression for the elements in $D_{\text {alcove }}$ (see $\S 2.2)$.

Theorem 3.3. Let $\sigma \in W_{f}^{+}$so that $\lambda^{\sigma} \in D_{\text {alcove }}$ (see §2.2). Then

$$
\lambda^{\sigma}=\sum_{\varphi \in \Delta_{+}} n_{\varphi}(\sigma) \varphi
$$

Proof. By definition $\hat{\rho}=d / 2+\rho$ (see (3.16)). Thus $2 \hat{\rho}=d+2 \rho$. On the other hand by the definition of $\bar{\sigma}$ (see (3.12), (3.13) and (3.15)) one has

$$
\begin{aligned}
\sigma(2 \rho) & =\zeta(\bar{\sigma}(d+2 \rho) \\
& =p(\bar{\sigma}(2 \hat{\rho})
\end{aligned}
$$


But $2 \rho=\zeta(2 \hat{\rho})$. But then

$$
\sigma(2 \rho)-2 \rho=\zeta(\bar{\sigma}(2 \hat{\rho})-\zeta(2 \hat{\rho})
$$

But $\bar{\sigma}$ is linear. Thus, by (3.19),

$$
\begin{aligned}
\sigma(2 \rho)-2 \rho & =2 \zeta(\bar{\sigma}(\hat{\rho})-\hat{\rho}) \\
& \left.=2 \sum_{\varphi \in \Delta_{+}} n_{\varphi}(\sigma) \varphi\right)
\end{aligned}
$$

But $\sigma(2 \rho)-2 \rho=2 \lambda^{\sigma}$ by (2.6). But then (3.19) follows from (3.22). QED

Remark 3.4.. As mentioned in the proof above $\bar{\sigma}$ is linear and we have used this fact. However $\sigma$ is not linear and (2.6) does not imply that $\sigma(\rho)-\rho=\lambda^{\sigma}$. In fact in general $\sigma(\rho)-\rho \neq \lambda^{\sigma}$. Instead one has $\sigma(2 \rho) / 2-\rho=\lambda^{\sigma}$ by $(2.6)$.

3.3. Recall (3.2). For notational simplicity put $\mathfrak{u}=t \mathfrak{g}[t]$ and $\mathfrak{u}^{-}=t^{-1} \mathfrak{g}\left[t^{-1}\right]$. To state the results of $[\mathrm{G}],[\mathrm{G}-\mathrm{L}]$ and $[\mathrm{Ku}-1,2]$ on the homology $H_{*}\left(\mathfrak{u}^{-}\right)$it is clarifying to write down a $\hat{\mathfrak{h}}$-weight basis of the exterior algebra $\wedge \mathfrak{u}^{-}$.

Let $J=\{1,2, \ldots, \operatorname{dim} \mathfrak{k}\}$ and let $x_{j}, j \in J$, be a $\mathfrak{h}$-weight basis of $\mathfrak{g}$ (under the adjoint representation). For any $j \in J$ let $\mu_{j} \in \mathfrak{h}$ be the weight corresponding to $x_{j}$ so that $\mu_{j} \in \Delta \cup\{0\}$. For any $n \in \mathbb{N}$ let $I_{n}=\{1, \ldots, n\}$ and $P_{n}$ be the set of all partitions $p=\left(p_{1}, \ldots, p_{n}\right)$ of length (exactly) $n$. Let $R_{n}$ be the set of all maps

$$
r: I_{n} \rightarrow \mathbb{N} \times J
$$

where if $r(i)=\left(r_{i}, r_{[i]}\right)$ then (1) $p(r)=\left(r_{1}, \ldots, r_{n}\right) \in P_{n}$ and (2) if $i<j$ and $r_{i}=r_{j}$ then

$$
r_{[i]}>r_{[j]}
$$

Given $r \in R_{n}$ let

$$
z_{r}=t^{-r_{1}} x_{r_{[1]}} \wedge \cdots \wedge t^{-r_{n}} x_{r_{[n]}}
$$

so that

$$
z_{r} \in \wedge^{n} \mathfrak{u}^{-}
$$

Let $\mu(r)=\sum_{i \in I_{n}} \mu_{r_{[i]}}$. The size, $\left|p_{r}\right|$, of the partition $p_{r}$ equals $\sum_{i \in I_{n}} r_{i}$. Since $d \in \hat{\mathfrak{h}}$ operates as $t \frac{d}{d t}$ one immediately has

Proposition 3.5. Let $r \in R_{n}$. Then $z_{r} \in \wedge^{n} \mathfrak{u}^{-}$is a $\hat{\mathfrak{h}}$-weight vector of weight

$$
-|p(r)| \delta+\mu(r)
$$

The condition (3.24) in the definition of $r$ guarantees that the elements $z_{r}, r \in R_{n}$, are linearly independent. In fact one immediately notes 
Proposition 3.6. The set $\left\{z_{r}\right\}, r \in R_{n}$, is a $\hat{\mathfrak{h}}$-weight basis $\wedge^{n} \mathfrak{u}^{-}$.

Let $R=\cup_{n \in \mathbb{Z}_{+}} R_{n}$ so that $\left\{z_{r}\right\}, r \in R$, is a $\hat{\mathfrak{h}}$-weight basis $\wedge \mathfrak{u}^{-}$. If $Y$ is any $\hat{\mathfrak{h}}$-module and $k \in \mathbb{Z}$ we will denote the eigensubspace of $Y$, with eigenvalue (t-weight) $-k$, for the action of $d$, by $(Y)_{k}$. Clearly $\left\{z_{r}\right\}, r \in R,|p(r)|=k$, is a basis of $\left(\wedge \mathfrak{u}^{-}\right)_{k}$. It is immediate from $(3.25)$ that $\left(\wedge \mathfrak{u}^{-}\right)_{k}$ is finite dimensional and one has the direct sum

$$
\wedge \mathfrak{u}^{-}=\sum_{k \in \mathbb{Z}_{+}}\left(\wedge \mathfrak{u}^{-}\right)_{k}
$$

3.4. Now $\mathfrak{u}^{-}$(and of course also $\mathfrak{u}$ ) is a Lie subalgebra of $\hat{\mathfrak{g}}$ and $\wedge \mathfrak{u}^{-}$is a chain complex for the Lie algebra homology space $H_{*}\left(\wedge \mathfrak{u}^{-}\right)$. Since $\mathfrak{g}_{\mathfrak{d}}$ (see $(3.2)$ ) obviously normalizes $\mathfrak{u}^{-}$one notes that $\wedge \mathfrak{u}^{-}$and $H_{*}\left(\wedge \mathfrak{u}^{-}\right)$are completely reducible $\mathfrak{g}_{\mathfrak{d}}$-modules with finite dimensional irreducible components. In fact since $d$ is central in $\hat{\mathfrak{g}}$ it is immediate that $\left(\wedge \mathfrak{u}^{-}\right)_{k}$ is a finite dimensional, completely reducible, $\mathfrak{g}$-module subcomplex (with homogeneous components $\left.\left(\wedge^{n} \mathfrak{u}^{-}\right)_{k}=\wedge^{n} \mathfrak{u}^{-} \cap\left(\wedge \mathfrak{u}^{-}\right)_{k}\right)$ and $\left(H_{*}\left(\wedge \mathfrak{u}^{-}\right)\right)_{k}$ is just the homology of $\left(\wedge \mathfrak{u}^{-}\right)_{k}$. The decomposition (3.26) yields the direct sum, $\mathfrak{g}$-module decomposition, with completely reducible finite dimensional components,

$$
H_{*}\left(\wedge \mathfrak{u}^{-}\right)=\sum_{k \in \mathbb{Z}_{+}}\left(H_{*}\left(\wedge \mathfrak{u}^{-}\right)\right)_{k}
$$

The determination of $H_{*}\left(\wedge \mathfrak{u}^{-}\right)$as a $\mathfrak{g}$-module is due to H. Garland. See Theorem 3.2 in [G]. This result was extended to the general Kac-Moody case (and, in addition, with values in a suitable module) in [G-L]. See theorem 8.5 in [G-L]. An elegant presentation of the Garland-Lepowsky theory is given in $\S 3.2$ of $[\mathrm{Ku}-2]$. The proof of Theorem 3.2 depends upon Theorem 2.5 in [G]. The latter (see Theorem 3.7 below), of interest in itself, is a statement about the Laplacian operator $L$ (denoted by $\Delta$ in [G]) associated to the boundary operator of $\wedge \mathfrak{u}^{-}$and a positive definite Hermitian structure on $\wedge \mathfrak{u}^{-}$. The operator $L$ commutes with the action of $\mathfrak{g}_{\mathfrak{d}}$ so that if $\operatorname{Har}\left(\mathfrak{u}^{-}\right)=\operatorname{Ker} L$ and $\operatorname{Har}_{n}\left(\mathfrak{u}^{-}\right)=\operatorname{Ker} L \mid \wedge^{n} \mathfrak{u}^{-}$then one has an isomorphism

$$
\operatorname{Har}\left(\mathfrak{u}^{-}\right) \equiv H_{*}\left(\wedge \mathfrak{u}^{-}\right)
$$

of graded $\mathfrak{g}_{\mathfrak{d}}$-modules. Thus it suffices to explicitly determine $L$ and its kernel. This determination rests upon Theorem 2.5 of [G]. As far as I am aware of, a proof of this theorem has not appeared in the literature. However the result is established as a special case of a much stronger theorem (arbitrary symmetrizable Kac-Moody case together with a suitable module) due to Kumar in [Ku-1]. Also see Theorem 3.4.2 in $[\mathrm{Ku}-2]$ and the final remark on p. 107 in $[\mathrm{Ku}-2]$.

If $\xi \in \hat{\mathfrak{h}}$ is an $\hat{\mathfrak{h}}$-weight occurring in $\wedge \mathfrak{u}^{-}$then $\xi=a \delta+\nu$ where $-a \in \mathbb{Z}_{+}$and $\nu$ is in the ordinary root lattice of $\mathfrak{h}$. We may refer to $a$ as the $\delta$ component of $\xi$ and $\nu$ as the $\mathfrak{h}$ component of $\xi$. The statement that $\xi$ is dominant is just the statement that $\nu \in D$. In particular if $\xi$ is the highest weight of a $\mathfrak{g}_{\delta}$ irreducible component of $\wedge \mathfrak{u}^{-}$ then certainly $\nu \in D$. 
Theorem 3.7 (Garland). Let $k \in \mathbb{Z}_{+}$and let $m_{k}$ be the maximal eigenvalue of Cas on $\left(\wedge \mathfrak{u}^{-}\right)_{k}$. Then

$$
m_{k} \leq k
$$

Furthermore one has equality in (3.29) if and only if $\left(\operatorname{Har}\left(\mathfrak{u}^{-}\right)\right)_{k} \neq 0$. Moreover in such a case $\left(\operatorname{Har}\left(\mathfrak{u}^{-}\right)\right)_{k}$ is the eigenspace in $\left(\wedge \mathfrak{u}^{-}\right)_{k}$ for Cas belonging to the eigenvalue $k$.

Proof (Kumar). Let $Z$ be an irreducible $\mathfrak{g}_{\mathfrak{d}}$-submodule of $\left(\wedge \mathfrak{u}^{-}\right)_{k}$ and let $\xi$ be the highest weight. Then $\xi=-k \delta+\nu$ for some $\nu \in D$. Then $L \mid Z$ operates as the scalar operator

$$
c=1 / 2((\hat{\rho}, \hat{\rho})-(\hat{\rho}-k \delta+\nu, \hat{\rho}-k \delta+\nu))
$$

by Theorem 3.4.2 in [Kum-2] since in the case at hand $\lambda=0$. But $\hat{\rho}=\rho+d / 2$ by (3.16). Thus $c=1 / 2((\rho, \rho)-(\rho+\nu, \rho+\nu)+k)$. That is $c=1 / 2(k-\operatorname{Cas}(\nu))$. But $L \mid Z$ is positive semidefinite. This implies the inequality (3.29). But $c=0$ if and only if $\operatorname{Cas}(\nu)=k$ and this must be the case if and only if $m_{k}=k$ and $Z \subset\left(\operatorname{Har}\left(\mathfrak{u}^{-}\right)\right)_{k}$. QED

Remark 3.8. Garland remarks that his Theorem 3.2 is an analogue of results in [Ko-1]. At first glance Theorem 2.5 in [G], upon which his Theorem 3.2 depends, appears to have no analogue in [Ko-1]. However Kumar's more general result, Theorem 3.4 .2 in [Ku-2], is in fact manifestly an infinite-dimensional analogue of Theorem 5.7 in $[\mathrm{Ko}-1]$.

The following statement is a corollary of Theorem 3.7.

Theorem 3.9. Let $0 \neq z$ be an $\hat{\mathfrak{h}}$-weight vector in $\wedge \mathfrak{u}^{-}$and let $Z \subset \wedge \mathfrak{u}^{-}$be the $\mathfrak{g}_{\mathfrak{0}}$-submodule generated by $z$. Let $\xi \in \hat{\mathfrak{h}}$ be the weight of $z$ and let $\lambda$ be the $\mathfrak{h}$-component of $\xi$. Then $Z \subset \operatorname{Har}\left(\wedge \mathfrak{u}^{-}\right)$and $z$ is a highest weight vector of $Z$ (so that $Z$ is an irreducible $\mathfrak{g}_{\mathfrak{o}}$-submodule) if and only if the $\delta$-component of $\xi$ equals $(\rho, \rho)-(\lambda+\rho, \lambda+\rho)$. Moreover in such a case $\lambda \in D$ and

$$
\xi=-\operatorname{Cas}(\lambda) \delta+\lambda
$$

Proof. Let $-k$ be the $\delta$-component of $\xi$ so that $k \in \mathbb{N}$ and $Z \subset\left(\wedge \mathfrak{u}^{-}\right)_{k}$. Now if $Z \subset \operatorname{Har}\left(\wedge \mathfrak{u}^{-}\right)$then $Z \subset\left(\operatorname{Har}\left(\wedge \mathfrak{u}^{-}\right)\right)_{k}$ and hence $\operatorname{Cas} \mid Z$ is the scalar operator for the scalar $k$, by Theorem 3.7. But if also $z$ is a highest weight vector of $Z$ then $\lambda \in D$ and

$$
k=(\lambda+\rho, \lambda+\rho)-(\rho, \rho)
$$

Conversely assume (3.31). If $z$ is not a highest weight vector of $Z$ there obviously exists an irreducible $\mathfrak{g}_{\mathfrak{d}}$-submodule $Z^{\prime} \subset Z$, necessarily having $\xi$ as a weight, and such that if $\xi^{\prime}=-k \delta+\lambda^{\prime}$ is the highest weight of $Z^{\prime}$ then $\lambda \neq \lambda^{\prime}$. But $\lambda^{\prime} \in D$ and $\operatorname{Cas}\left(\lambda^{\prime}\right)=\left(\lambda^{\prime}+\rho, \lambda^{\prime}+\rho\right)-(\rho, \rho)$. But then $\operatorname{Cas}\left(\lambda^{\prime}\right)>k$ by (3.31) and the 
Freudenthal result (5.9.2) in [Ko-1]. This contradicts (3.29). Thus $z$ is a highest weight vector of $Z$ and $\lambda \in D$. But then the right hand side of (3.31) equals $\operatorname{Cas}(\lambda)$. Hence $Z \subset \operatorname{Har}\left(\mathfrak{u}^{-}\right)$by Theorem 3.7. QED

3.5. Theorem 3.2 in $[\mathrm{G}]$ gives the decomposition of $\operatorname{Har}\left(\wedge \mathfrak{u}^{-}\right)$(and hence equivalently $\left.H_{*}\left(\wedge \mathfrak{u}^{-}\right)\right)$as a $\mathfrak{g}_{\mathfrak{d}}$-module. This result (see Theorem 3.10 below) is also the application of Theorem 8.5 in [G-L] to the case of the standard maximum parabolic subalgebra of the affine Kac-Moody Lie algebra where the module is trivial. See also Theorem 3.2.7 in [Ku-2] for this case. The statement is made stronger (implicit in [G-L]) by including Lemma 3.2.6 in [Ku-2]. One notes that the strenthened statement is a Kac-Moody analogue of Lemma 5.12 and Theorem 5.14 in [Ko-1]. By (3.18) one has

$$
\bar{\sigma}(\hat{\rho})-\hat{\rho}=-\left\langle\Phi_{\bar{\sigma}}\right\rangle
$$

for any $\sigma \in W_{f}$.

Theorem 3.10 (Garland). Let $\sigma \in W_{f}^{+}$. Then $-\left\langle\Phi_{\bar{\sigma}}\right\rangle$ occurs as a $\mathfrak{h}_{\delta}$-weight of multiplicity one in the $\mathfrak{g}_{\mathfrak{d}}$ - module $\wedge \mathfrak{u}^{-}$. In particular there exists a unique $r \in R$ (see §3.3), henceforth denoted by $r^{\sigma}$, such that $z_{r^{\sigma}}$ is a weight vector with weight $-\left\langle\Phi_{\bar{\sigma}}\right\rangle$. In the notation of Lemma 3.5

$$
-\left\langle\Phi_{\bar{\sigma}}\right\rangle=-\left|p\left(r^{\sigma}\right)\right| \delta+\mu\left(r^{\sigma}\right)
$$

In addition $-\left\langle\Phi_{\bar{\sigma}}\right\rangle$ is dominant. That is, $\mu\left(r^{\sigma}\right) \in D$.

Let $Z_{\sigma}$ be the $\mathfrak{g}_{\mathfrak{d}}$-module generated by $z_{r^{\sigma}}$. Then $Z_{\sigma}$ is $\mathfrak{g}_{\mathfrak{d}}$-irreducible and $z_{r^{\sigma}}$ is a highest weight vector of $Z_{\sigma}$. Moreover $Z_{\sigma} \subset \operatorname{Har}\left(\wedge \mathfrak{u}^{-}\right)$and indeed one has the multiplicity free decomposition

$$
\operatorname{Har}\left(\wedge \mathfrak{u}^{-}\right)=\sum_{\sigma \in W_{f}^{+}} Z_{\sigma}
$$

With respect to the two compatible gradations, $\operatorname{Har}_{n}\left(\wedge \mathfrak{u}^{-}\right)$and $\left(\operatorname{Har}\left(\wedge \mathfrak{u}^{-}\right)\right)_{k}$ one has

$$
\left(\operatorname{Har}\left(\wedge \mathfrak{u}^{-}\right)\right)_{k}=\sum_{\sigma \in W_{f}^{+},\left|p\left(r^{\sigma}\right)\right|=k} Z_{\sigma}
$$

and

$$
\operatorname{Har}_{n}\left(\wedge \mathfrak{u}^{-}\right)=\sum_{\sigma \in W_{f}^{+}, \ell(\sigma)=n} Z_{\sigma}
$$

Remark 3.11. Note that the existence of $r^{\sigma}$ with the cited properties is a consequence of Proposition 3.6 and the multiplicity one property of $-\left\langle\Phi_{\bar{\sigma}}\right\rangle$. Note also that $\left(\operatorname{Har}\left(\wedge \mathfrak{u}^{-}\right)\right)_{k}$ is finite dimensional since $\left(\wedge \mathfrak{u}^{-}\right)_{k}$ is finite dimensional (see $\S 3.3$ ). On the other hand $\operatorname{Har}_{n}\left(\wedge \mathfrak{u}^{-}\right)$is also finite dimensional (even though $\wedge^{n} \mathfrak{u}$ is infinite dimensional) since the set $\left\{\sigma \in W_{f} \mid \ell(\sigma)=n\right\}$ is obviously finite. 
3.6. Remark 3.12. Since the set $E=\left\{t^{-i} x_{j} \mid(i, j) \in \mathbb{N} \times J\right\}$ is clearly a basis of $\mathfrak{u}^{-}$note that the elements $z_{S_{k}}, k \in I_{n}$, are linearly independent in $\wedge \mathfrak{u}^{-}$where $S_{k}, k \in I_{n}$, are finite mutually distinct subsets of $E$ and for any such subset $S, z_{S}$ is the decomposable element obtained by exterior multiplication, in some order, of the elements in $S$.

Recalling the notation of (3.23) let $J^{+}=\left\{j \in J \mid \mu_{j} \in \Delta_{+}\right\}$. We may choose the ordering of the basis $\left\{x_{j}\right\}$ of $\mathfrak{g}$ so that $J^{+}=\{1, \ldots, m\}$ where here $m=$ card $\Delta_{+}$. For $j \in J^{+}$we now write $\varphi_{j}$ for $\mu_{j}$ and choose $x_{j}=e_{\varphi_{j}}$.

For $n \in \mathbb{Z}_{+}$let $R_{n}^{+}$be the set of all $r \in R_{n}$ such that the image of (3.23) is contained in $\mathbb{N} \times J^{+}$. Now let $\sigma \in W_{f}^{+}$and let $n=\ell(\sigma)$. For $j \in J^{+}$let $n_{\varphi_{j}}(\sigma)$ be defined as in $\S 1.3$ and let $z^{\sigma, j} \in \wedge^{n_{\varphi_{j}}(\sigma)} \mathfrak{u}^{-}$be defined by putting $z^{\sigma, j}=1$ if $n_{\varphi_{j}}(\sigma)=0$ and otherwise

$$
z^{\sigma, j}=t^{-1} x_{j} \wedge \cdots \wedge t^{-n_{\varphi_{j}}(\sigma)} x_{j}
$$

Next let $z^{\sigma} \in \wedge^{n} \mathfrak{u}^{-}$(see (1.6)) be defined by putting

$$
z^{\sigma}=z^{\sigma, 1} \wedge \cdots \wedge z^{\sigma, m}
$$

We now use the results of $\S 3.2$ and relate Theorem 3.10 with $D_{\text {alcove }}$. See $\S 2.2$ and the cautionary Remark 3.4.

Theorem 3.13. Let $\sigma \in W_{f}^{+}$and let $\lambda^{\sigma} \in D_{\text {alcove }}$ be defined as in (2.5). Then in the notation of (3.33) one has $\mu\left(r^{\sigma}\right)=\lambda^{\sigma}$ and in fact (3.33) can be written

$$
-\left\langle\Phi_{\bar{\sigma}}\right\rangle=-\operatorname{Cas}\left(\lambda^{\sigma}\right) \delta+\lambda^{\sigma}
$$

In particular not only is $\operatorname{Cas}\left(\lambda^{\sigma}\right)$ an integer but in fact

$$
\operatorname{Cas}\left(\lambda^{\sigma}\right)=\sum_{\varphi \in \Delta_{+}} n_{\varphi}(\sigma)\left(n_{\varphi}(\sigma)+1\right) / 2
$$

Moreover (recalling (3.38) and Theorem 3.10) one has $r^{\sigma} \in R_{n}^{+}$and

$$
z_{r^{\sigma}}= \pm z^{\sigma}
$$

Finally $Z_{\sigma} \equiv V_{\lambda^{\sigma}}$ as a $\mathfrak{g}$-module and, as a $\mathfrak{g}$-module, $\operatorname{Har}\left(\wedge \mathfrak{u}^{-}\right)$is multiplicity free and one has the equivalence

$$
\operatorname{Har}\left(\wedge \mathfrak{u}^{-}\right) \equiv \sum_{\sigma \in W_{f}^{+}} V_{\lambda^{\sigma}}
$$

Of course the same statement is true when $H_{*}\left(\wedge \mathfrak{u}^{-}\right)$replaces $\operatorname{Har}\left(\wedge \mathfrak{u}^{-}\right)$. 
Proof. The statement that $\mu\left(r^{\sigma}\right)=\lambda^{\sigma}$ is immediate from (3.19),(3.20),(3.32) and (3.33). Since $z_{r^{\sigma}}$ and $Z_{\sigma}$ of Theorem 3.10 satisfies the condition of $z$ and $Z$ of Theorem 3.9 it follows from (3.30) that the $\delta$ component of $-\left\langle\Phi_{\bar{\sigma}}\right\rangle$ equals $-\operatorname{Cas}\left(\lambda^{\sigma}\right)$. This proves (3.39). But then (3.40) follows from (3.19). But now the $\hat{\mathfrak{h}}$ weight of the weight vector $z^{\sigma}$ is clearly

$$
\left(-\sum_{j=1}^{m} n_{\varphi_{j}}(\sigma)\left(n_{\varphi_{j}}(\sigma)+1\right) / 2\right) \delta+\sum_{j=1}^{m} n_{\varphi_{j}}(\sigma) \varphi_{j}
$$

Thus $z^{\sigma}$ is an $\hat{\mathfrak{h}}$ weight vector of weight $-\left\langle\Phi_{\bar{\sigma}}\right\rangle$ by (3.19). Thus one has (3.41) by the multiplicity one condition (Theorem 3.10) of this weight in $\wedge \mathfrak{u}^{-}$. It follows in particular, (see Remark 3.12) that $r^{\sigma} \in R_{n}^{+}$. The remaining statements are then obvious noting that the $\mathfrak{h}$ component of $-\left\langle\Phi_{\bar{\sigma}}\right\rangle$ determines the $\delta$-component (or using the injectivity of the map (2.8)). QED

3.7. Let $Q=\left(\mathbb{Z}_{+}\right)^{m}$ where $m=\operatorname{card} \Delta_{+}$. If $q \in Q$ let $q_{i} \in \mathbb{Z}_{+}, i \in I_{m}$, be defined so that $q=\left(q_{1}, \ldots, q_{m}\right)$. Let $X \subset \mathfrak{h}$ be the semigroup generated by $\Delta_{+}$and let $\eta: Q \rightarrow X$ be defined by putting

$$
\eta(q)=\sum_{i \in I_{m}} q_{i} \varphi_{i}
$$

If $\eta(q)=\mu$ we will refer to $q$ as a positive root partition of $\mu$. For any $\mu \in X$ let

$$
Q_{\mu}=\eta^{-1}(\mu)
$$

so that $Q_{\mu}$ is the set of all positive root partitions of $\mu$.

Now if $q \in Q$ let

$$
c(q)=\sum_{i \in I_{m}} q_{i}\left(q_{i}+1\right) / 2
$$

and let $z^{(q)} \in \wedge \mathfrak{u}^{-}$be defined by putting

$$
z^{(q)}=z^{(q), 1} \wedge \cdots \wedge z^{(q), m}
$$

where $z^{(q), i}=1$ if $q_{i}=0$ and otherwise

$$
z^{(q), i}=t^{-1} x_{i} \wedge \cdots \wedge t^{-q_{i}} x_{i}
$$

One notes that $z^{(q)}$ is an $\hat{\mathfrak{h}}$-weight vector and the $\hat{\mathfrak{h}}$

$$
\text { weight of } z^{(q)}=-c(q) \delta+\eta(q)
$$

Let $\sigma \in W_{F}^{+}$. Then by (3.20) one has $\lambda^{\sigma} \in X$, i.e. $D_{\text {alcove }} \subset X$, and in addition (3.20) defines a distinguished positive root partition of $\lambda^{\sigma}$. We denote this partition 
by $q^{\sigma}$ and refer to this partition as the $\sigma$ positve root partition of $\lambda^{\sigma}$. Thus $q^{\sigma} \in Q_{\lambda^{\sigma}}$ is given by $q_{i}^{\sigma}=n_{\varphi_{i}}(\sigma)$ for all $i \in I_{m}$, using the notation of $\S 1.3$. The following result characterizes the elements in the subset $D_{\text {alcove }} \subset X$ and for, each $\sigma \in W_{f}^{+}$, the result characterizes the $\sigma$ positive root partition among all the positive root partitions in $Q_{\lambda^{\sigma}}$.

Theorem 3.14. Let $q \in Q$. Then

$$
c(q) \geq(\mu+\rho, \mu+\rho)-(\rho, \rho)
$$

where $\mu=\eta(q)$. Furthermore one has equality in (3.48) if and only if $\mu=\lambda^{\sigma}$ for some (necessarily unique, see (2.8)) $\sigma \in W_{f}^{+}$and $q=q^{\sigma}$.

Proof. Let $k=c(q)$ so that $Z \subset\left(\wedge \mathfrak{u}^{-}\right)_{k}$ where $Z$ is $\mathfrak{g}_{\mathfrak{d}}$-submodule generated by $z^{(q)}$. But then, using the notation of (3.29), one readily has, using e.g. the Freudenthal result (5.9.2) in [Ko-1], $(\mu+\rho, \mu+\rho)-(\rho, \rho) \leq m_{k}$. But $m_{k} \leq k$ by (3.29). This establishes the inequality (3.38).

Now if $\mu=\lambda^{\sigma}$ and $q=q^{\sigma}$ one has equality in (3.48) by (3.40). Conversely if one has equality in (3.48) then, by Theorem $3.9, Z$ is a $\mathfrak{g}_{\mathfrak{d}}$-irreducible component of $\operatorname{Har}\left(\wedge \mathfrak{u}^{-}\right)$and $z^{(q)}$ is a highest weight vector. Then by Theorems 3.10 and 3.13 there exists $\sigma \in W_{f}^{+}$such that the highest weight of $Z$ is $-\operatorname{Cas}\left(\lambda^{\sigma}\right) \delta+\lambda^{\sigma}$ and this weight occurs with multiplicity 1 in $\wedge \mathfrak{u}^{-}$. But then $z^{(q)}=z^{\sigma}$ (up to scalar multiplication). But then $q=q^{\sigma}$ by Remark 3.12. QED

3.8. Using, in the present context, notation introduced in $\S 5.1$ of [Ko-1], one defines an operation $\dot{+}$, referred to as root addition, on the set of all subsets of the set of affine roots. If $\Psi_{i} \subset \hat{\Delta}^{\prime}, i=1,2$, then $\Psi=\Psi_{1} \dot{+} \Psi_{2}$ if $\Psi=\left\{\beta \in \hat{\Delta}^{\prime} \mid \beta=\right.$ $\beta_{1}+\beta_{2}$, for some $\left.\beta_{i} \in \Psi_{i}\right\}$. Let $\Psi \subset \hat{\Delta}^{\prime}$. We will say that Psi is closed under root addition if $\Psi \dot{+} \Psi=\Psi$ and $\Psi$ is abelian or commutative if $\Psi \dot{+} \Psi=\emptyset$. A subset $\Phi \subset \Delta_{+}$is called ideal in $\Delta_{+}$if $\Delta_{+} \dot{+} \Phi \subset \Phi$. If $\Phi_{i}, i=1,2$, are two such ideals then obviously $\Phi_{1}+\Phi_{2}$ is again such an ideal.

Let $\sigma \in W_{f}^{+}$and let $L(\sigma)=\max _{\varphi \in \Delta_{+}} n_{\varphi}(\sigma)$ using the notation of $\S 1.3$. Since $\psi$ is the highest weight of the adjoint representation, clearly (see Remark 1.2)

$$
n_{\psi}(\sigma) \geq n_{\varphi}(\sigma)
$$

for any $\varphi \in \Delta_{+}$. Thus

$$
L(\sigma)=n_{\psi}(\sigma)
$$

which, in the notation of $\S 1.4$ implies that

$$
A_{\sigma} \subset \mathfrak{h}^{(L(\sigma)+1)} \text { but } A_{\sigma} \not \subset \mathfrak{h}^{(L(\sigma))}
$$

In Theorem 3.16 below we observe that $\sigma$ defines a chain of $L(\sigma)+1$ ideals (not necessarily distinct) of $\Delta_{+}$. For any $i \in \mathbb{Z}_{+}$let

$$
\Delta_{i}(\sigma)=\left\{\varphi \in \Delta_{+} \mid i \leq n_{\varphi}(\sigma)\right\}
$$


so that $\Delta_{i}(\sigma)=\emptyset$ if $i>L(\sigma)$. Clearly

$$
\Delta_{L(\sigma)}(\sigma) \subset \cdots \subset \Delta_{0}(\sigma)=\Delta_{+}
$$

Remark 3.15. Observe that if $i \in \mathbb{N}, \varphi \in \Delta_{+}$and $\sigma \in W_{f}^{+}$then, by Proposition 3.1 ,

$$
\varphi \in \Delta_{i}(\sigma) \Longleftrightarrow i \delta-\varphi \in \Phi_{\bar{\sigma}}
$$

(Note the exclusion of $i=0$ ).

If $\varphi_{1}, \varphi_{2} \in \Delta_{+}$and $\varphi_{1}+\varphi_{2}$ is a root then note that by Remark 1.2 one has

$$
n_{\varphi_{1}+\varphi_{2}}(\sigma) \in\left\{n_{\varphi_{1}}(\sigma)+n_{\varphi_{2}}(\sigma), n_{\varphi_{1}}(\sigma)+n_{\varphi_{2}}(\sigma)+1\right\}
$$

for any $\sigma \in W_{f}^{+}$.

Theorem 3.16. Let $\sigma \in W_{f}^{+}$. Then $\Delta_{i}(\sigma)$ is an ideal in $\Delta_{+}$for any $i \in \mathbb{Z}_{+}$. Furthermore

$$
\Delta_{i}(\sigma) \dot{+} \Delta_{j}(\sigma) \subset \Delta_{i+j}(\sigma)
$$

for any $i, j \in \mathbb{Z}_{+}$so that in particular $\Delta_{L(\sigma)}(\sigma)$ is an abelian ideal in $\Delta_{+}$. Finally using the notation of $\S 3.2$ one has

$$
\lambda^{\sigma}=\sum_{i \in I_{L(\sigma)}}\left\langle\Delta_{i}(\sigma)\right\rangle
$$

Proof. The first statement and (3.55) are immediate consequences of (3.54). On the other hand (3.56) clearly follows from (3.20) and (3.53). QED

\section{The main results}

4.1. We recall results in the 1965 paper [Ko-2]. Let $\mathfrak{u} \subset \mathfrak{g}$ be any (complex) subspace. Let $k=\operatorname{dim} \mathfrak{u}$ so that $\wedge^{k} \mathfrak{u}$ is a 1-dimensional subspace of $\wedge^{k} \mathfrak{g}$. Let $M$ be the maximal dimension of an abelian subalgebra of $\mathfrak{g}$. For any $k \in \mathbb{Z}_{+}$let $C_{k} \subset \wedge^{k} \mathfrak{g}$ be the span of all 1-dimensional subspaces of the form $\wedge^{k} \mathfrak{a}$ where $\mathfrak{a} \subset \mathfrak{g}$ is a $k$ dimensional abelian subalgebra of $\mathfrak{g}$. Obviously $C_{k}=0$ if $k>M$. Clearly $C_{k}$ is a $\mathfrak{g}$-submodule of $\wedge^{k} \mathfrak{g}$ under the adjoint action of $\mathfrak{g}$. Of course the same is true of

$$
C=\sum_{\mathfrak{k}=0}^{M} C_{k}
$$

If a (complex) subspace $\mathfrak{u} \subset \mathfrak{g}$ is stable under $\mathfrak{h}$ let $\Delta(\mathfrak{u})=\left\{\varphi \in \Delta \mid e_{\varphi} \in \mathfrak{u}\right\}$. Let $\mathfrak{b}$ be the Borel subalgebra of $\mathfrak{g}$ containing $\mathfrak{h}$ such that $\Delta(\mathfrak{b})=\Delta_{+}$and let $\mathfrak{n}=[\mathfrak{b}, \mathfrak{b}]$ 
be the nilradical of $\mathfrak{b}$. Let $\Xi$ be an index set parameterizing the set of all abelian ideals in $\mathfrak{b}$ and for any $\xi \in \Xi$ let $\mathfrak{a}_{\xi}$ be the corresponding abelian ideal. For any $\xi \in \Xi$ it is immediate that $\mathfrak{a}_{\xi} \subset \mathfrak{n}$ so that $\Xi$ is finite and

$$
\mathfrak{a}_{\xi}=\sum_{\varphi \in \Delta\left(\mathfrak{a}_{\xi}\right)} \mathbb{C} e_{\varphi}
$$

and hence

$$
\wedge^{k} \mathfrak{a}_{\xi}=\mathbb{C} e_{\varphi_{1}} \wedge \cdots \wedge e_{\varphi_{k}}
$$

where $k=\operatorname{dim} \mathfrak{a}_{\xi}$ and

$$
\Delta\left(\mathfrak{a}_{\xi}\right)=\left\{\varphi_{1}, \ldots, \varphi_{k}\right\}
$$

The subsets of $\Delta_{+}$of the form (4.3) are characterized by Theorem (8) in [Ko-2]. For $k \in \mathbb{Z}_{+}$where $k \leq \operatorname{card} \Delta_{+}$let $\Xi_{k}=\left\{\xi \in \Xi \mid \operatorname{dim} \mathfrak{a}_{\xi}=k\right\}$. Of course $\Xi_{k}$ is empty if $k>M$. If $\mu \in \mathfrak{h}$ let $|\mu|=(\mu, \mu)^{\frac{1}{2}}$. The characterization is as follows:

Theorem 4.1. Let $\Phi \subset \Delta_{+}$and let $k=\operatorname{card} \Phi$. Then if $\Phi=\left\{\varphi_{1}, \ldots, \varphi_{k}\right\}$ one has

$$
\left|\rho+\varphi_{1}+\cdots+\varphi_{k}\right|^{2}-|\rho|^{2} \leq k
$$

and one has equality in (4.4) if and only if there exists $\xi \in \Xi_{k}$ (necessariy unique) such that $\Phi=\Delta\left(\mathfrak{a}_{\xi}\right)$.

The adjoint action of $\mathfrak{g}$ on itself induces the structure of a $\mathfrak{g}$-module (and hence $U(\mathfrak{g})$-module) on $\wedge \mathfrak{g}$. Let $\xi \in \Xi$. Since $\left[\mathfrak{b}, \mathfrak{a}_{\xi}\right] \subset \mathfrak{a}_{\xi}$ it is immediate from (4.2) that, if $\xi \in \Xi_{k}$, then $\wedge^{k} \mathfrak{a}_{\xi} \subset \wedge^{k} \mathfrak{g}$ is a highest weight space and hence, under the action of $\mathfrak{g}$, generates an irreducible $\mathfrak{g}$-submodule, denoted here by $V_{\xi}$, of $\wedge^{k} \mathfrak{g}$. Furthermore if we put

$$
\lambda_{\xi}=\sum_{\varphi \in \Delta\left(\mathfrak{a}_{\xi}\right)} \varphi
$$

then $\lambda_{\xi}$ is the highest weight of $V_{\xi}$. Theorem (7) in [Ko-2] implies that, for $\xi, \xi^{\prime} \in \Xi$,

$$
\lambda_{\xi}=\lambda_{\xi^{\prime}} \Longleftrightarrow \xi=\xi^{\prime}
$$

This accounts for the multiplicity-free statement in the following result (Theorem 4.2). Like Theorem 4.1, Theorem 4.2 is also part of Theorem (8) in [Ko-2].

Theorem 4.2. For any $\mathfrak{k} \in \mathbb{Z}_{+}$where $0 \leq k \leq M$ one has the direct sums

$$
C_{k}=\sum_{\xi \in \Xi_{k}} V_{\xi}
$$

and

$$
C=\sum_{\xi \in \Xi} V_{\xi}
$$


Furthermore $C$ and, a fortiori, $C_{k}$, are multiplicity-free $\mathfrak{g}$-modules.

4.2. It is a beautiful result of Dale Peterson that $\operatorname{card} \Xi=2^{\ell}$. Although Peterson's proof has not been published a sketch of his proof appears in $\S 2$ of [Ko-5]. A key (and, for me, surprising) point of the proof was the connection established between $\Xi$ and a subset of $W_{f}^{+}$. Expanding on this connection P. Cellini and P. Papi published a simpler proof of Peterson's theorem in [C-P]. See Theorem 2.9 in that reference. Recalling the notation of $\S 1.4$ in our present paper here let $W_{f}^{(k)}=\left\{\sigma \in W_{f}^{+} \mid A_{\sigma} \subset \mathfrak{h}^{(k)}\right\}$. By Proposition 1.4 one has card $W_{f}^{(2)}=2^{\ell}$. Peterson's theorem follows from a bijection

$$
\Xi \rightarrow W_{f}^{(2)}
$$

established in $[\mathrm{C}-\mathrm{P}]$.

Remark 4.3. Note that if $\sigma \in W_{f}^{+}$then

$$
\sigma \in W_{f}^{(2)} \Longleftrightarrow n_{\varphi}(\sigma) \in\{0,1\}, \forall \varphi \in \Delta_{+}
$$

Indeed by definition $\sigma \in W_{f}^{(2)}$ if and only if $n_{\psi}(\sigma) \in\{0,1\}$. But then (4.9) follows from (3.49).

Recently Ruedi Suter in [Su] showed (again a surprise for me) showed that Peterson's theorem, in fact, follows from a result in [Ko-2]. In more detail, Theorem 4.4, below, was known to me before [Su]. In fact it is an immediate consequence of Peterson's Proposition 2.5 in [Ko-5] and the result (4.8) in [C-P]. The equation (4.10) below was discovered independently by Suter in $[\mathrm{Su}]$. But the main novelty is that the proof of $(4.10),(4.11)$ and hence (4.13) in [Su] depends only on a 1965 result in [Ko-2], stated in the present paper as Theorem 4.1. With the benefit of this knowledge we will prove Theorem 4.4 using only [Ko-2] and results established in the present paper.

Theorem 4.4. For any $\xi \in \Xi$ there exists an (necessarily unique) element $\sigma_{\xi} \in$ $W_{f}^{+}$such that

$$
\lambda_{\xi}=\lambda^{\sigma_{\xi}}
$$

Moreover $\sigma_{\xi} \in W_{f}^{(2)}$ and the map

$$
\xi \rightarrow W_{f}^{(2)}, \quad \xi \mapsto \sigma_{\xi}
$$

is a bijection. In particular one has the inclusion

$$
\left\{\lambda_{\xi} \mid \xi \in \Xi\right\} \subset D_{\text {alcove }}
$$

and the count (Peterson's theorem)

$$
\operatorname{card} \Xi=2^{\ell}
$$


Proof. Let $\xi \in \Xi$. Then, in the notation of $\S 3.7,(4.5)$ is a root partition $q$ of $\lambda$ (see (3.43)) and $q_{i} \in\{0,1\}$ for any $i \in I_{m}$. But then if $\xi \in \Xi_{k}$ one has $c(q)=k$ (see (3.45)). But then $c(q)=\left|\lambda_{\xi}+\rho\right|^{2}-\left.\rho\right|^{2}$ by Theorem 4.1. Thus there exists an element $\sigma_{\xi} \in W_{f}^{+}$satisfying (4.10) and $q=q^{\sigma_{\xi}}$ by Theorem 3.14. Obviously if $\varphi \in \Delta_{+}$then

$$
n_{\varphi}\left(\sigma_{\xi}\right)=1 \text { if } \varphi \in \Delta\left(\mathfrak{a}_{\xi}\right) \text { and } n_{\varphi}\left(\sigma_{\xi}\right)=0 \text { if } \varphi \notin \Delta\left(\mathfrak{a}_{\xi}\right)
$$

But then $\sigma_{\xi} \in W_{f}^{(2)}$ by (4.9). The map (4.11) is injective by (4.6).

Conversely let $\sigma \in W_{f}^{(2)}$. Without loss we may assume that $\sigma \neq 1$. Then by (4.9) and Theorem 3.16 the set

$$
\Phi=\left\{\varphi \in \Delta_{+} \mid n_{\varphi}(\sigma)=1\right\}
$$

is an abelian ideal in $\Delta_{+}$. Thus there exists $\xi \in \Xi$ such that $\lambda_{\xi}=\langle\Phi\rangle$. On the other hand $\lambda^{\sigma}=\langle\Phi\rangle$ by (3.20). Hence $\sigma=\sigma_{\xi}$. Consequently (4.11) is surjective. QED

As a consequence of Remark 4.3 and Theorem 4.4 one establishes the following property of $W_{f}^{(2)}$.

Theorem 4.5. Let $\sigma \in W_{f}^{+}$. Then

$$
\operatorname{Cas}\left(\lambda^{\sigma}\right) \geq \ell(\sigma)
$$

and equality occurs in (4.16) if and only if $\sigma \in W_{f}^{(2)}$. Furthermore in that case writing $\sigma=\sigma_{\xi}$ for $\xi \in \Xi$ (Theorem 4.4) one has

$$
\begin{aligned}
\operatorname{Cas}\left(\lambda^{\sigma}\right) & =\ell(\sigma) \\
& =\operatorname{dim} \mathfrak{a}_{\xi}
\end{aligned}
$$

Proof. The inequality (4.16) follows from (1.6) and (3.40). But then equality occurs if and only if $n_{\varphi}(\sigma) \in\{0,1\}$ for all $\varphi \in \Delta_{+}$. But this is the case if and only if $\sigma \in W_{f}^{(2)}$ by Remark 4.3. The final statement then follows from Theorem 4.4 and (4.14). QED

4.3. Recall §3.3. Define a pairing of $\mathfrak{u}^{-}$and $\mathfrak{u}=t \mathfrak{g}[t]$ so that for $p, q \in \mathbb{N}$ and $x, y \in \mathfrak{g}$ then $\left(t^{-p} x, t^{q} y\right)=0$ if $p \neq q$ and $\left(t^{-p} x, t^{p} y\right)=(x, y)$. Let $y_{k}, k \in J$ be the basis of $\mathfrak{g}$, dual to the $x_{j}$, so that $y_{k}$ is a weight vector of weight $-\mu_{k}$. It follows then that $\left\{t^{q} y_{k} \mid(q, k) \in \mathbb{N} \times J\right\}$ is an $\hat{\mathfrak{h}}$ basis of $\mathfrak{u}$, dual to the basis $\left\{t^{-p} x_{j} \mid(p, j) \in \mathbb{N} \times J\right\}$ of $\mathfrak{u}^{-}$. We may identify $\mathfrak{u}^{-}$here with the subspace of all linear functionals $f$ on $\mathfrak{u}$ which vanish on $t^{N} \mathfrak{g}$ (where $N$ depends on $f$ ) for sufficiently large $N$.

If $r$ is defined as in (3.23) let $w_{r} \in \wedge^{n} \mathfrak{u}$ be defined so that

$$
w_{r}=t^{r_{1}} y_{r_{[1]}} \wedge \cdots \wedge t^{r_{n}} y_{r_{[n]}}
$$


The obvious analogue of Proposition 3.5 and 3.6 is

Proposition 4.6. Let $r \in R_{n}$. Then $w_{r}$ is a $\hat{\mathfrak{h}}$-weight vector of weight

$$
p(r) \delta-\mu(r)
$$

and the set $\left\{w_{r} \mid r \in R_{n}\right\}$ is a basis of $\wedge^{n} \mathfrak{u}$.

The pairing of $\mathfrak{u}^{-}$and $\mathfrak{u}$ extends, as usual (determinently), to a nonsingular pairing of $\wedge \mathfrak{u}^{-}$and $\wedge \mathfrak{u}$. The subspaces $\wedge^{m} \mathfrak{u}^{-}$and $\wedge^{n} \mathfrak{u}$ are orthogonal if $m \neq n$ and if $n=m$ one notes that $\left\{w_{r} \mid r \in R_{n}\right\}$ and $\left\{z_{r} \mid r \in R_{n}\right\}$ are dual bases. The pairing of $\wedge \mathfrak{u}^{-}$and $\wedge \mathfrak{u}$ is clearly invariant under the action of $\mathfrak{g}_{\mathfrak{o}}$. If $y \in \mathfrak{u}$ let $\theta(y) \in E n d \wedge \mathfrak{u}$ be the operator of the adjoint action of $y$ on $\wedge \mathfrak{u}$ and if $x \in \mathfrak{u}^{-}$let $\iota(x) \in E n d \wedge \mathfrak{u}$ be the interior product by $x$. Let $\partial_{+}$be the boundary operator on $\wedge \mathfrak{u}$ whose derived homology is $H_{*}(\mathfrak{u})$. Using a standard expression for $\partial_{+}$one has that if $u \in \wedge \mathfrak{u}$ then

$$
\partial_{+} u=1 / 2 \sum_{(p, j) \in \mathbb{N} \times J} \theta\left(t^{p} y_{j}\right) \iota\left(t^{-p} x_{j}\right) u
$$

noting that $\iota\left(t^{-p} x_{j}\right) u$ is nonzero for only a finite subset of $\mathbb{N} \times J$. Let $\theta^{*}\left(t^{p} y_{j}\right) \in$ $\operatorname{End}(\wedge \mathfrak{u})^{*}$ be the negative transpose of $\theta\left(t^{p} y_{j}\right)$. It is immediate that $\wedge \mathfrak{u}^{-}$is stable under $\theta^{*}\left(t^{p} y_{j}\right)$ and that, using the notation of $(3.26)$,

$$
\theta^{*}\left(t^{p} y_{j}\right):\left(\wedge \mathfrak{u}^{-}\right)_{k} \rightarrow\left(\wedge \mathfrak{u}^{-}\right)_{k-p}
$$

noting $\left(\wedge \mathfrak{u}^{-}\right)_{j}=0$ if $j$ is negative. For any $x \in \mathfrak{u}^{-}$let $\varepsilon(x) \in E n d \wedge \mathfrak{u}^{-}$be the operator of exterior multiplication by $x$. One sees that if $d_{+} \in \operatorname{End}(\wedge \mathfrak{u})^{*}$ is the negative transpose of $\partial_{+}$then $\wedge \mathfrak{u}^{-}$is stable under $d_{+}$and, in fact for any $v \in \wedge \mathfrak{u}^{-}$ one has

$$
d_{+} v=1 / 2 \sum_{(p, j) \in \mathbb{N} \times J} \varepsilon\left(t^{-p} x_{j}\right) \theta^{*}\left(t^{p} y_{j}\right) v
$$

noting that, by $(4.20), \theta^{*}\left(t^{p} y_{j}\right) v \neq 0$ for only a finite subset of $\mathbb{N} \times J$. Of course the pair $\left((\wedge \mathfrak{u})^{*}, d_{+}\right)$is the cochain complex whose derived cohomology is $H^{*}(\mathfrak{u})$. The pair $\left(\wedge \mathfrak{u}^{-}, d_{+}\right)$is a subcomplex and we denote the derived cohomology by $\mathcal{H}^{*}(\mathfrak{u})$. Since $d_{+}$is antiderivation in either case both $H^{*}(\mathfrak{u})$ and $\mathcal{H}^{*}(\mathfrak{u})$ are algebras and one has an algebra homomorphism

$$
\mathcal{H}^{*}(\mathfrak{u}) \rightarrow H^{*}(\mathfrak{u})
$$

On the other hand (see $\S 3.4) \wedge \mathfrak{u}^{-}$has a bigrading $\left(\wedge^{n} \mathfrak{u}^{-}\right)_{k}$ and one notes from $(4.21)$ that

$$
d_{+}:\left(\wedge^{n} \mathfrak{u}^{-}\right)_{k} \rightarrow\left(\wedge^{n+1} \mathfrak{u}^{-}\right)_{k}
$$

In particular $\left(\left(\wedge \mathfrak{u}^{-}\right)_{k}, d_{+}\right)$(see $\S 3.4$.) is a finite dimensional $\mathfrak{g}_{\mathfrak{d}}$-completely reducible subcomplex of $\left(\wedge \mathfrak{u}^{-}, d_{+}\right)$, for $k \in \mathbb{Z}_{+}$. The derived cohomology is denoted by $\mathcal{H}(\mathfrak{u})_{k}$. 
Since the $r_{i}$ in (3.25) are positive one notes that $\left(\wedge^{n} \mathfrak{u}^{-}\right)_{k}=0$ for $n>k$. Also note that (see (3.25)) for $n, n^{\prime}, k, k^{\prime} \in \mathbb{Z}_{+}$,

$$
\left(\wedge^{n} \mathfrak{u}^{-}\right)_{k} \wedge\left(\wedge^{n^{\prime}} \mathfrak{u}^{-}\right)_{k^{\prime}} \subset\left(\wedge^{n+n^{\prime}} \mathfrak{u}^{-}\right)_{k+k^{\prime}}
$$

Of course $\wedge$ induces cup product $(\vee)$ in $\mathcal{H}(\mathfrak{u})$. This establishes

Proposition 4.7. One has the direct sum

$$
\mathcal{H}(\mathfrak{u})=\sum_{k \in \mathbb{Z}_{+}}(\mathcal{H}(\mathfrak{u}))_{k}
$$

where $(\mathcal{H}(\mathfrak{u}))_{k}$ is a finite dimensional $\mathfrak{g}_{\mathfrak{d}}$-completely reducible $\mathfrak{g}_{\mathfrak{d}}$-module. Furthermore with regard to cohomological degree

$$
(\mathcal{H}(\mathfrak{u}))_{k}=\sum_{n=0}^{k}\left(\mathcal{H}^{n}(\mathfrak{u})\right)_{k}
$$

and one has the cup product relation

$$
\left(\mathcal{H}^{n}(\mathfrak{u})\right)_{k} \vee\left(\mathcal{H}^{n^{\prime}}(\mathfrak{u})\right)_{k^{\prime}} \subset\left(\mathcal{H}^{n+n^{\prime}}(\mathfrak{u})\right)_{k+k^{\prime}}
$$

4.4. In $\S 3.4$ we introduced Garland's harmonic subspace $\operatorname{Har}\left(\wedge \mathfrak{u}^{-}\right)$of $\wedge \mathfrak{u}^{-}$. The subspace $\operatorname{Har}\left(\wedge \mathfrak{u}^{-}\right)$is a space of cycles for the boundary operator on $\wedge \mathfrak{u}^{-}$and the quotient map $\operatorname{Har}\left(\wedge \mathfrak{u}^{-}\right) \rightarrow H_{*}\left(\mathfrak{u}^{-}\right)$is an isomorphism (see (3.28)). We will now see that $\operatorname{Har}\left(\mathfrak{u}^{-}\right)$also represents the cohomology $\mathcal{H}(\mathfrak{u})$. We will first clarify the structure of $\operatorname{Har}\left(\wedge \mathfrak{u}^{-}\right)$. For any $\sigma \in W_{f}^{+}$we have defined a $\mathfrak{g}_{\mathfrak{o}}$-irreducible submodule $Z_{\sigma}$ of $\wedge \mathfrak{u}^{-}$ in (Garland) Theorem 3.10. Combining Theorem 3.10 and Theorem 3.13 one has

Theorem 4.8. The highest weight of $Z_{\sigma}$ is $-\operatorname{Cas}\left(\lambda^{\sigma}\right) \delta+\lambda^{\sigma}$. Furthermore this weight occurs with multiplicity 1 in $\wedge \mathfrak{u}^{-}$so that, a fortiori, the representation of $\mathfrak{g}_{\mathfrak{0}}$ afforded by $Z_{\sigma}$ occurs with multiplicity 1 in $\wedge \mathfrak{u}^{-}$. Next

$$
Z_{\sigma} \subset\left(\wedge^{\ell(\sigma)}\left(\mathfrak{u}^{-}\right)\right)_{C a s \lambda^{\sigma}}
$$

In fact

$$
\left(\operatorname{Har}_{n}\left(\wedge \mathfrak{u}^{-}\right)\right)_{k}=\sum_{\sigma \in W_{f}^{+}, \ell(\sigma)=n, \operatorname{Cas}\left(\lambda^{\sigma}\right)=k} Z_{\sigma}
$$

If $\lambda \in D$ then, as one knows, the dual $\left(V_{\lambda}\right)^{*}$, as a $\mathfrak{g}$-module, is characterized by the property that $-\lambda$ is the lowest weight of $\left(V_{\lambda}\right)^{*}$. A similar statement is clearly true 
for the reductive Lie algebra $\mathfrak{g}_{\mathfrak{d}}$. As a consequence of Theorem 4.8 one can then make the following

Remark 4.9. If $\sigma \in W_{f}^{+}$then the dual $\left(Z_{\sigma}\right)^{*}$, as a $\mathfrak{g}_{\mathfrak{0}}$-module, is characterized by the property that $\operatorname{Cas}\left(\lambda^{\sigma}\right) \delta-\lambda^{\sigma}$ is the lowest weight of $\left(Z_{\sigma}\right)^{*}$.

Now one knows that there exists an automorphism $\theta$ on $\mathfrak{g}$ which stabilizes $\mathfrak{h}$ and is such that $\theta \mid \mathfrak{h}$ is minus the identity. In particular $\theta\left(\Delta_{+}\right)=-\Delta$ so that if $\kappa \in W$ is the long element then $\kappa \theta$ stabilizes $\Delta_{+}$and also stabilizes $D$. If $\lambda \in D$ let $\lambda^{\prime}=\kappa \theta(\lambda)$. Since the $-\lambda$ is the lowest weight of $V_{\lambda^{\prime}}$ we may identify $V_{\lambda^{\prime}}$ with the dual $\mathfrak{g}$-module $V_{\lambda}^{*}$.

Remark 4.10. As an application of Theorem 2.4 note that $D_{\text {alcove }}$ is stable under $\kappa \theta$. Indeed since $\chi_{\lambda}\left(a_{P}\right) \in\{-1,1\}$ clearly (by the reality of the character value) $\chi_{\lambda^{\prime}}\left(a_{P}\right)=\chi_{\lambda}\left(a_{P}\right)$ so that $\chi_{\lambda^{\prime}}\left(a_{P}\right) \in\{-1,1\}$. Thus there exists an involutory bijection $W_{f}^{+} \rightarrow W_{f}^{+}, \quad \sigma \mapsto \sigma^{\prime}$ such that

$$
\left(\lambda^{\sigma}\right)^{\prime}=\lambda^{\sigma^{\prime}}
$$

Examples exist where $\sigma^{\prime} \neq \sigma$.

The automorphism $\theta$ clearly extends to an automorphism of $\hat{\mathfrak{g}}$ which stabilizes $\hat{\mathfrak{h}}$ and is minus the identity on $\hat{\mathfrak{h}}$. But then $\theta$ interchanges $\mathfrak{u}$ and $\mathfrak{u}^{-}$since $\theta(\delta)=$ $-\delta$. As noted in Remark 4.9, for $\sigma \in W_{f}^{*}$, the irreducible $\mathfrak{g}_{\mathfrak{d}}$-module with lowest weight $\operatorname{Cas}\left(\lambda^{\sigma}\right) \delta-\lambda^{\sigma}$ readily identifies with $\left(Z_{\sigma}\right)^{*}$, the dual $\mathfrak{g}_{\mathfrak{d}}$-module to $Z_{\sigma}$. But now Theorem 3.10 and Theorem 3.12 determines the $\mathfrak{g}_{\mathfrak{d}}$-module structure of $H_{*}(\mathfrak{u})$. Recalling the notation at the end of $\S 3.3$ one has the direct sum

$$
H_{*}(\mathfrak{u})=\sum_{k \in \mathbb{Z}_{+}}\left(H_{*}(\mathfrak{u})\right)_{-k}
$$

Furthermore $\left(H_{*}(\mathfrak{u})\right)_{-k}$ is a finite dimensional completely reducible $\mathfrak{g}_{\mathfrak{d}}$-module and as such

$$
\left(H_{*}(\mathfrak{u})\right)_{-k} \equiv \sum_{\sigma \in W_{f}^{+}, \operatorname{Cas}\left(\lambda^{\sigma}\right)=k}\left(Z_{\sigma}\right)^{*}
$$

We can now prove that $\operatorname{Har}\left(\wedge \mathfrak{u}^{-}\right) \subset \wedge \mathfrak{u}^{-}$represents the cohomology $\mathcal{H}(\mathfrak{u})$ as well as the homology (see (3.28)) $H_{*}\left(\mathfrak{u}^{-}\right)$.

Theorem 4.11. Any element in $\operatorname{Har}\left(\wedge \mathfrak{u}^{-}\right)$is a $d_{+}$-cocycle and the induced linear map

$$
\operatorname{Har}\left(\wedge \mathfrak{u}^{-}\right) \rightarrow \mathcal{H}^{*}(\mathfrak{u})
$$

is a $\mathfrak{g}_{\mathfrak{d}}$-module isomorphism. In particular (4.32) restricts to an isomorphism

$$
\left(\operatorname{Har}\left(\wedge \mathfrak{u}^{-}\right)\right)_{k} \rightarrow\left(\mathcal{H}^{*}(\mathfrak{u})\right)_{k}
$$


of finite dimensional completely reducible $\mathfrak{g}_{\mathfrak{d}}$-modules, for any $k \in \mathbb{Z}_{+}$.

Proof. It clearly suffices to prove (4.33). But the nonsingular pairing of $\wedge \mathfrak{u}^{-}$ and $\wedge \mathfrak{u}$ induces a nonsingular pairing of the finite dimensional completely reducible $\mathfrak{g}_{0}$ modules $\left(\wedge \mathfrak{u}^{-}\right)_{k}$ and $(\wedge \mathfrak{u})_{-k}$. But $d_{+} \mid\left(\wedge \mathfrak{u}^{-}\right)_{k}$ is the negative transpose of $\partial_{+} \mid(\wedge \mathfrak{u})_{-k}$. Thus, as $\mathfrak{g}_{\mathfrak{d}}$-modules one has

$$
\left(\mathcal{H}^{*}(\mathfrak{u})\right)_{k} \equiv \sum_{\sigma \in W_{f}^{+}, \operatorname{Cas}\left(\lambda^{\sigma}\right)=k} Z_{\sigma}
$$

by (4.31). But by complete reducibility and the multiplicity 1 statement in Theorem 4.8 one must have $\left.\operatorname{Har}_{n}\left(\wedge \mathfrak{u}^{-}\right)\right)_{k} \subset \operatorname{Kerd}_{+}$(see (4.28)) and the $\mathfrak{g}_{\mathrm{d}}$-isomorphism (4.33). QED

4.5. We now introduce a new grading $\mathcal{H}^{[j]}(\mathfrak{u})$ on $\mathcal{H}^{*}(\mathfrak{u})$. In doing so we are following a suggestion of Pavel Etingof who pointed out to us that our subsequent results can be neatly formulated using this grading. As noted in $\S 4.3$ one has $\left(\wedge^{n} \mathfrak{u}^{-}\right)_{k}=0$ for $n>k$. In particular $\left(\mathcal{H}^{n}(\mathfrak{u})\right)_{k}=0$ for $n>k$ (see (4.25)). For $j \in \mathbb{Z}$ let

$$
\wedge^{[j]} \mathfrak{u}^{-}=\sum_{n, k \in \mathbb{Z}_{+}, k-n=j}\left(\wedge^{n} \mathfrak{u}\right)_{k}
$$

and let

$$
\mathcal{H}^{[j]}(\mathfrak{u})=\sum_{n, k \in \mathbb{Z}_{+}, k-n=j}\left(\mathcal{H}^{n}(\mathfrak{u})\right)_{k}
$$

so that one has the direct sums

$$
\begin{aligned}
\wedge \mathfrak{u}^{-} & =\sum_{j \in \mathbb{Z}_{+}} \wedge^{[j]} \mathfrak{u} \\
\mathcal{H}^{*}(\mathfrak{u}) & =\sum_{j \in \mathbb{Z}_{+}} \mathcal{H}^{[j]}(\mathfrak{u})
\end{aligned}
$$

Proposition 4.12. The subspaces $\wedge^{[j]} \mathfrak{u}^{-}$and, a fortiori, $\mathcal{H}^{[j]}(\mathfrak{u})$ are finite dimensional. Moreover, with respect to wedge and cup product

$$
\begin{aligned}
& \left(\wedge^{[j]} \mathfrak{u}^{-}\right) \wedge\left(\wedge^{\left[j^{\prime}\right]} \mathfrak{u}^{-}\right) \subset\left(\wedge^{\left[j+j^{\prime}\right]} \mathfrak{u}^{-}\right) \\
& \mathcal{H}^{[j]}(\mathfrak{u}) \vee \mathcal{H}^{\left[j^{\prime}\right]}(\mathfrak{u}) \subset \mathcal{H}^{\left[j+j^{\prime}\right]}(\mathfrak{u})
\end{aligned}
$$

In particular $\wedge^{[0]} \mathfrak{u}^{-}$is a finite dimensional subalgebra of $\wedge \mathfrak{u}^{-}$and $\mathcal{H}^{(0)}(\mathfrak{u})$ is a finite dimensional subalgebra of $\mathcal{H}^{*}(\mathfrak{u})$.

Proof. Clearly (4.37) follows from (4.23). It suffices only to show that $\left(\wedge^{[j]} \mathfrak{u}^{-}\right)$ is finite dimensional for any $j \in \mathbb{Z}_{+}$. Recalling the notation of $\S 3.3$ note that, for any $r \in R_{n}$,

$$
z_{r} \in \wedge^{[|p(r)|-n]} \mathfrak{u}^{-}
$$


But, by (3.24), the number of $i \in I_{n}$ such that $r_{i}=1$ is at most $\operatorname{dim} \mathfrak{k}$ for any $n$. This implies that $|p(r)|-n \geq n-\operatorname{dim} \mathfrak{k}$. But this readily implies that $\wedge^{[j]} \mathfrak{u}^{-}$is finite dimensional, for any $j \in \mathbb{Z}_{+}$. QED

Remark 4.13. Note that as a $\mathfrak{g}_{\mathfrak{d}}$-module one has

$$
\mathcal{H}^{[j]}(\mathfrak{u}) \equiv \sum_{\sigma \in W_{f}^{+}, \operatorname{Cas}\left(\lambda^{\sigma}\right)-\ell(\sigma)=j} Z_{\sigma}
$$

Indeed (4.39) follows from (3.33), (3.35), (3.36) and (3.39).

Now note that by (4.22) one has

$$
d_{+}: \wedge^{[j]} \mathfrak{u}^{-} \rightarrow \wedge^{[j-1]} \mathfrak{u}^{-}
$$

for any $j \in \mathbb{Z}$. This implies part of

Lemma 4.14. One has

$$
\wedge^{[0]} \mathfrak{u}^{-} \subset \operatorname{Ker} d_{+}
$$

Moreover as an algebra and a $\mathfrak{g}_{\mathfrak{d}}$ module one has $\wedge^{[0]} \mathfrak{u}^{-} \equiv \wedge \mathfrak{g}$. In fact there exists a $\mathfrak{g}_{\mathfrak{d}}$-module algebra isomorphism

$$
\wedge \mathfrak{g} \rightarrow \wedge^{[0]} \mathfrak{u}^{-}
$$

where, if $u_{i} \in \mathfrak{g}, i=1, \ldots, k$, then

$$
u_{1} \wedge \cdots \wedge u_{k} \mapsto t^{-1} u_{1} \wedge \cdots \wedge t^{-1} u_{k}
$$

Proof. One has (4.41) by (4.40) since of course $\wedge^{[-1]} \mathfrak{u}^{-}=0$. Recalling (3.25) the condition that $z_{r} \in \wedge^{[0]} \mathfrak{u}^{-}$is that $r_{i}=1$ for all $i \in I_{n}$. But this clearly implies that there is a $\mathfrak{g}_{\mathfrak{d}}$-module algebra isomorphism (4.42) satisfying (4.43). QED

Now if $u \in \mathfrak{g}$ then in the notation of (4.21)), clearly $\theta^{*}\left(t^{p} y_{j}\right)\left(t^{-2} u\right)=0$ if $p \geq 2$ (since $\wedge \mathfrak{u}^{-}$is stable, by definition, under $\theta^{*}\left(t^{p} y_{j}\right)$ ) and

$$
\varepsilon\left(t^{-1} x_{j}\right) \theta^{*}\left(t^{1} y_{j}\right)\left(t^{-2} u\right)=t^{-1} x_{j} \wedge t^{-1}\left[y_{j}, u\right]
$$

Hence

$$
d_{+}\left(t^{-2} u\right)=\sum_{j \in J} t^{-1} x_{j} \wedge t^{-1}\left[y_{j}, u\right]
$$

We have identified $\mathfrak{g}$ with its dual $\mathfrak{g}^{*}$ using the Killing form so that if $d$ is the CartanEilenberg-Koszul coboundary operator on $\wedge \mathfrak{g}^{*}$ it, with this identification, is an antiderivation of degree 1 in $\wedge \mathfrak{g}$. Using the standard formula for $d$ one then has $d u \in \wedge^{2} \mathfrak{g}$ for any $u \in \mathfrak{g}$ and explicitly

$$
d u=1 / 2 \sum_{j \in J} x_{j} \wedge\left[y_{j}, u\right]
$$


Let $(d \mathfrak{g})$ be the ideal in $\wedge \mathfrak{g}$ generated by $d \mathfrak{g}$. In $\S 4.1$ we introduced the multiplicityfree $\mathfrak{g}$-submodule $C \subset \wedge \mathfrak{g}$ defined by the set of all abelian subalgebras of $\mathfrak{g}$. Theorem 4.2 asserts that the highest weight vectors in $C$ are given by the $2^{\ell}$ abelian ideals in $\mathfrak{b}$. Theorem 4.3. in [Ko-6] contains the following statement

Theorem 4.15. One has the direct sum

$$
C \oplus(d \mathfrak{g})
$$

The space $C$ inherits an algebra structure, as a consequence of Theorem 4.15, since (4.46) implies that

$$
C \equiv \wedge \mathfrak{g} /(d \mathfrak{g})
$$

At the time [Ko-6] was written I had no idea about the meaning of this algebra structure. This question is resolved in the following theorem (Theorem 4.16). We could use Theorem 4.15 to prove much of Theorem 4.16. However, we will, instead, prove Theorem 4.16 using results established in the present paper.

The advantage in dealing with cohomology $\mathcal{H}^{*}(\mathfrak{u})$ instead of homology $H_{*}\left(\mathfrak{u}^{-}\right)$ is that $\mathcal{H}^{*}(\mathfrak{u})$ has an algebra stucture. The nature of this algebra is, nevertheless, presently, quite mysterious to us. However for the finite dimensional subalgebra $\mathcal{H}^{[0]}(\mathfrak{u})$ one has the following result.

Theorem 4.16. As a $\mathfrak{g}$-module $\mathcal{H}^{[0]}(\mathfrak{u})$ is multiplicity-free with $2^{\ell}$ irreducible components. In fact (recalling $§ 4.1$ )

$$
\mathcal{H}^{[0]}(\mathfrak{u}) \equiv \sum_{\xi \in \Xi} V_{\xi}
$$

As an algebra (under cup product)

$$
\mathcal{H}^{[0]}(\mathfrak{u}) \equiv \wedge \mathfrak{g} /(d \mathfrak{g})
$$

Proof. The statement (4.48) follows from (4.39) and Theorem 4.5.

Now by (4.40) and (4.41) one has

$$
\mathcal{H}^{[0]}=\wedge^{[0]} \mathfrak{u}^{-} / d_{+}\left(\wedge^{[1]} \mathfrak{u}^{-}\right)
$$

But $z_{r} \in \wedge^{[1]} \mathfrak{u}^{-}$, by (3.25), if and only if all but one $r_{i}=1$ and the remaining $r_{i}$ equals 2 . Thus

$$
\wedge^{[1]} \mathfrak{u}^{-}=\left(\wedge^{[0]} \mathfrak{u}^{-}\right) \wedge t^{-2} \mathfrak{g}
$$

But then $d_{+}\left(\wedge^{[1]} \mathfrak{u}^{-}\right)$is the ideal in $\wedge^{[0]} \mathfrak{u}^{-}$generated by $d_{+}\left(t^{-2} \mathfrak{g}\right)$, by (4.41). The algebra isomorphism (4.49) then follows immediately from (4.42), (4.43), (4.44) and (4.45). QED 
Recall the notation of $\S 4.1$. If $k \in \mathbb{Z}_{+}$then we have defined $C_{k} \subset \wedge^{k} \mathfrak{g}$ in terms of all the abelian subalgebras of $\mathfrak{g}$ having dimension $k$. Theorem (5) in [Ko-2] gives a different characterization of $C_{k}$. Note that, by (2.1.7) in [Ko-2], the Laplacian $L$ is Cas $/ 2$ operating in $\wedge \mathfrak{g}$. For any $k \in \mathbb{Z}_{+}$let $m_{(k)}$ be the maximal eigenvalue of Cas in $\wedge^{k} \mathfrak{g}$. Theorem (5) in [Ko-2] then asserts

Theorem 4.17. For any $k \in \mathbb{Z}_{+}$one has

$$
m_{(k)} \leq k
$$

Furthermore equality occurs in (4.52) if and only if $k \leq M$ in which case $C_{k}$ is the eigenspace for Cas (operating in $\wedge^{k} \mathfrak{g}$ ) corresponding to the eigenvalue $k$.

Remark 4.18. In the light of Lemma 4.14 and (4.48) it is not difficult to show that the 1965 result, Theorem 4.17 above, is, in fact, implied by Theorem 2.5 in Garland's 1975 paper [Gar].

4.6. Returning to (2.9) let $b_{k} \in \mathbb{Z}$, for $k \in \mathbb{Z}_{+}$, be defined so that

$$
\left(\prod_{n=1}^{\infty}\left(1-x^{n}\right)\right)^{\operatorname{dim} K}=\sum_{k \in \mathbb{Z}_{+}} b_{k} x^{k}
$$

By (2.9) and (2.10) one has

$$
\sum_{k \in \mathbb{Z}_{+}} b_{k} x^{k}=\sum_{\sigma \in W_{f}^{+}}(-1)^{\ell(\sigma)} \operatorname{dim} V_{\lambda^{\sigma}} x^{\operatorname{Cas}\left(\lambda^{\sigma}\right)}
$$

which immediately yields the finite sum (see (4.16))

$$
\begin{aligned}
b_{k} & =\sum_{\sigma \in W_{f}^{+}, \operatorname{Cas}\left(\lambda^{\sigma}\right)=k}(-1)^{\ell(\sigma)} \operatorname{dim} V_{\lambda^{\sigma}} \\
& =\sum_{\sigma \in W_{f}^{+}, \operatorname{Cas}\left(\lambda^{\sigma}\right)=k}(-1)^{\ell(\sigma)} \operatorname{dim} Z_{\sigma}
\end{aligned}
$$

Note that the second equality in (4.55) follows from the first line in Theorem 4.8.

Let $\mathfrak{v} \subset \mathfrak{n}$ be the span of $\left\{e_{\varphi} \mid(\psi, \varphi)>0\right\}$. One knows that $\mathfrak{v}$ is a Heisenberg Lie algebra so that we can write $\operatorname{dim} \mathfrak{v}=2 m+1$ where $m \in \mathbb{Z}_{+}$. One has $\mathbb{C} e_{\psi}=$ cent $\mathfrak{v}$. Here we are regarding the case where $m=0$ (i.e. when $\mathfrak{g} \equiv \operatorname{Lie} S l(2, \mathbb{C}$ ) as a Heisenberg Lie algebra. From the Heisenberg structure of $\mathfrak{v}$ one knows that there exists a partition $\Delta(\mathfrak{v})-\{\psi\}=\Delta^{1}(\mathfrak{v}) \cup \Delta^{2}(\mathfrak{v})$ where each of the two parts has $m$ roots which can be ordered so that if $\Delta^{1}(\mathfrak{v})=\left\{\beta_{1}, \ldots, \beta_{m}\right\}$ and $\Delta^{2}(\mathfrak{v})=\left\{\gamma_{1}, \ldots, \gamma_{m}\right\}$ then for $i=1, \ldots, m$,

$$
\beta_{i}+\gamma_{i}=\psi
$$


If $\mathfrak{g}$ is simply laced (A-D-E case) then we have known for some time that $m=h-2$ where $h$ is the Coxeter number. See e.g. (1.10.1), p. 214 in [Ko-4]. In the non-simply laced case we had checked that $m \geq \ell-1$. But one could do better. D. Peterson informed us that $m=h^{\vee}-2$ in general where $h^{\vee}$ is the dual Coxeter number (see $\S 1.5$ and the notational change in Proposition 1.8). Knowing this one readily supplies an easy proof.

Proposition 4.19. If $m$ is defined as in (4.56) then $m=h^{\vee}-2$ where $h^{\vee}$ is the dual Coxeter number (see $§ 1.5)$.

Proof. If $\varphi \in \Delta_{+}$then $(\varphi, \psi) \geq 0$ since $\psi$ is the highest root. But if $\varphi \in(\Delta(\mathfrak{v})-$ $\{\psi\})$ then $(\varphi, \psi)=(\psi, \psi) / 2$ since $\psi$ is a long root. Thus $(2 \rho, \psi)-(\psi, \psi)=m(\psi, \psi)$. Hence $(2 \rho, \psi)+(\psi, \psi)=(m+2)(\psi, \psi)$. But $(2 \rho, \psi)+(\psi, \psi)=1$ by $(1.18)$. But then $m=h^{\vee}-2$ by $(1.17)$. QED

We can now prove

Theorem 4.20. Let $\sigma$ lie in the complement of $W_{f}^{(2)}$ in $W_{f}^{+}$(see §4.2). Then

$$
\ell(\sigma) \geq h^{\vee}
$$

where $h^{\vee}$ is the dual Coxeter number (see $\left.\left.§ 1.5\right)\right)$.

Proof. By assumption, if $r^{\sigma} \in \operatorname{Reg} A_{\sigma}$ (see $\S 1.2$ ), one has $\psi\left(r^{\sigma}\right)>2$. But then, using (4.56) and Proposition 4.19, for any $i=1, \ldots, h^{\vee}-2$, one must have either $\beta_{i}\left(r^{\sigma}\right)>1$ or $\gamma_{i}\left(r^{\sigma}\right)>1$ and possibly both inequalities. In any case the number of $\varphi$-walls, where $\varphi \in \Delta(\mathfrak{u})-\{\psi\}$, separating $r^{\sigma}$ and $2 \rho$ is at least $h^{\vee}-2$. But since $\psi\left(r^{\sigma}\right)>2$, the walls $\mathfrak{h}_{\psi, 1}$ and $\mathfrak{h}_{\psi, 2}$ also separate $r^{\sigma}$ and $2 \rho$. This accounts for $h^{\vee}$ separating walls. This proves (4.57) (see (1.6)). QED

As a corollary one has

Theorem 4.21. Let $\sigma \in W_{f}^{+}$. If $\operatorname{Cas}\left(\lambda^{\sigma}\right) \leq h^{\vee}$, where $h^{\vee}$ is the dual Coxeter number (see §1.5), then $\sigma \in W_{f}^{(2)}$ (see §4.2)) and

$$
\operatorname{Cas}\left(\lambda^{\sigma}\right)=\ell(\sigma)
$$

Proof. If $\operatorname{Cas}\left(\lambda^{\sigma}\right)<h^{\vee}$ then $\ell(\sigma)<h^{\vee}$ by (4.16). Hence $\sigma \in W_{f}^{(2)}$ by Theorem 4.20. If $\operatorname{Cas}\left(\lambda^{\sigma}\right)=h^{\vee}$ then $\ell(\sigma) \leq h^{\vee}$ by (4.16). But if $\ell(\sigma)<h^{\vee}$ one has $\sigma \in W_{f}^{(2)}$ by Theorem 4.20. On the other hand if $\ell(\sigma)=h^{\vee}$ then one has the equality $\ell(\sigma)=$ $\operatorname{Cas}\left(\lambda^{\sigma}\right)$. But this implies that $\sigma \in W_{f}^{(2)}$ by Theorem 4.5. In any case $\sigma \in W_{f}^{(2)}$. But then one has (4.58) by Theorem 4.5. QED 
Let $P(t)$ be the power series given by (1.15) and defined by Bott, so that if $P(t)=\sum_{k=0}^{\infty} p_{k} t^{k}$ then $p_{k}$ is the $2 k$ Betti number of the loop group $\Omega(K)$. Another consequence of Theorem 4.20 is that we can count the number of abelian ideals in $\mathfrak{b}$ whose dimension is $k$ when $k<h^{\vee}$. Recall the notation of $\S 4.1$.

Theorem 4.22. If $k<h^{\vee}$ then

$$
\operatorname{card} \Xi_{k}=p_{k}
$$

where $p_{k}$ is the $2 k$ Betti number of the loop group $\Omega(K)$ and is given by (1.15).

Proof. By (1.14) and (1.15) $p_{k}$ is the number of alcoves $A_{\sigma}, \sigma \in W_{f}^{+}$, in $\mathfrak{h}^{+}$such that $\ell(\sigma)=k$. But if $k<h^{\vee}$ then, by Theorem 4.20, the set $\left\{\sigma \in W_{f}^{+} \mid \ell(\sigma)<h^{\vee}\right\}$ is contained in $W_{f}^{(2)}$. But then (4.59) follows from Theorem 4.3 and (4.17). QED

Our main results concern $b_{k}$ (see (4.53)) when $k \leq h^{\vee}$. One major difficulty in using (4.55) to determine $b_{k}$ is the cancelation in the sums of (4.55) due to the alternation signs. When $k \leq h^{\vee}$ this alternation disappears. The grading in $\wedge \mathfrak{g}$ induces a grading in the quotient algebra $\wedge \mathfrak{g} /(d \mathfrak{g})$.

$$
\wedge \mathfrak{g} /(d \mathfrak{g})=\sum_{k=0}^{M} \wedge^{k} \mathfrak{g} /(d \mathfrak{g})^{k}
$$

where $(d \mathfrak{g})^{k}=(d \mathfrak{g}) \cap \wedge^{k} \mathfrak{g}$. The following theorem is one our main results.

Theorem 4.23. Assume $k \leq h^{\vee}$. Then the following seven numbers are all equal
[1] $(-1)^{k} b_{k}$
[2] $\operatorname{dim} C_{k}$
[3] $\sum_{\xi \in \Xi_{k}} \operatorname{dim} V_{\xi}$
[4] $\operatorname{dim}\left\{v \in \wedge^{k} \mathfrak{g} \mid \theta\right.$ (Cas) $\left.v=k v\right\}$
[5] $\operatorname{dim} \wedge^{k} \mathfrak{g} /(d \mathfrak{g})^{k}$
[6] $\operatorname{dim} \mathcal{H}^{k}(\mathfrak{u})$
[7] $\operatorname{dim} H_{k}\left(\mathfrak{u}^{-}\right)$
$($ see $(4.53))$
(see $\$ 4.1)$
(see §4.2)
(see [Ko-2])
(see (4.47))
(see §4.3)
(see §3.4)

Proof. By Theorem 4.21 one may replace the upper sum in (4.55) by

$$
b_{k}=(-1)^{k} \sum_{\sigma \in W_{f}^{(2)}, \operatorname{Cas}\left(\lambda^{\sigma}\right)=k} \operatorname{dim} V_{\lambda^{\sigma}}
$$

But then recalling the definition of $\Xi_{k}$ in $\S 4.1$ and $V_{\xi}$ in $\S 4.2$ it follows from Theorem 4.3 and (4.17) that

$$
b_{k}=(-1)^{k} \sum_{\xi \in \Xi_{k}} \operatorname{dim} V_{\xi}
$$


This implies the equality of [1] and [3]. But then [2] and [3] are equal by (4.7). But [2] and [4] are equal by Theorem 4.17. One has the equality of [2] and [5] by (4.47). But both $\mathcal{H}^{k}(\mathfrak{u})$ and $H_{k}\left(\mathfrak{u}^{-}\right)$are in bijective correspondence with $\operatorname{Har}_{k}\left(u_{-}\right)$by Theorem 4.11 and (3.28). In particular one has a linear isomorphism $\mathcal{H}^{k}(\mathfrak{u}) \rightarrow H_{k}\left(\mathfrak{u}^{-}\right)$. This it suffices to prove the equality of $[6]$ and $[5]$. But

$$
\operatorname{Har}_{k}\left(\mathfrak{u}^{-}\right)=\sum_{\sigma \in W_{f}^{+}, \ell(\sigma)=k} Z_{\sigma}
$$

by (4.28). But if $\ell(\sigma)=k$ then $\sigma \in W_{f}^{(2)}$ by Theorem 4.20 and hence $\operatorname{Cas}\left(\lambda^{\sigma}\right)=k$ by Theorem 4.5. Thus

$$
\begin{aligned}
\mathcal{H}^{k}(\mathfrak{u}) & =\left(\mathcal{H}^{k}(\mathfrak{u})_{k}\right. \\
& \subset \mathcal{H}^{[0]}(\mathfrak{u})
\end{aligned}
$$

But then one has the equality of $[6]$ and $[5]$ by (4.49). QED

Example. Consider the case when $K=S U(5)$. Then $\operatorname{dim} \mathfrak{k}=24$ so that $b_{k}=\tau(k+1)$ where, using the terminology and notation of $\S 4.5$, Chapter 7 , in [Se], $n \mapsto \tau(n)$ is the Ramanujan tau function. In this case $h^{\vee}=5$ and choosing, say [2] in Theorem 4.23, the first 5 nontrivial Ramanujan numbers (see p. 97 in [Se] and also [L]) yield the equality

$$
\begin{aligned}
\operatorname{dim} C_{1} & =24 \\
\operatorname{dim} C_{2} & =252 \\
\operatorname{dim} C_{3} & =1472 \\
\operatorname{dim} C_{4} & =4870 \\
\operatorname{dim} C_{5} & =6048
\end{aligned}
$$

We thank P. Etingof for pointing out to us that Theorem 4.23 (and its proof) yield the acyclity of the complex (4.65) in Theorem 4.24 when $k \leq h^{\vee}$. Let $\partial_{-}$be the boundary operator in $\wedge \mathfrak{u}$ whose derived homology is $H_{*}\left(\mathfrak{u}^{-}\right)$. Then, as noted in $\S 3.4$, if $k \in \mathbb{Z}_{+},\left(\left(\wedge \mathfrak{u}^{-}\right)_{k}, \partial_{-}\right)$is a finite dimensional subcomplex of $\left(\left(\wedge \mathfrak{u}^{-}\right)_{k}, \partial_{-}\right)$. The subcomplex is described by the $\partial_{-}$-maps

$$
\left(\wedge^{k} \mathfrak{u}^{-}\right)_{k} \longrightarrow\left(\wedge^{k-1} \mathfrak{u}^{-}\right)_{k} \longrightarrow \cdots \longrightarrow\left(\wedge^{0} \mathfrak{u}^{-}\right)_{k} \longrightarrow 0
$$

(Here we are using the fact that $\left(\wedge^{n} \mathfrak{u}^{-}\right)_{k}=0$ if $n>k$. The latter statement is immediate from (3.25) and Proposition 3.6)

Theorem 4.24. If $k \leq h^{\vee}$ then the complex (4.65) is acyclic. That is, $\left(H_{n}\left(\mathfrak{u}^{-}\right)\right)_{k}=$ 0 unless $n=k$ so that $\left(H_{*}\left(\mathfrak{u}^{-}\right)\right)_{k}=\left(H_{k}\left(\mathfrak{u}^{-}\right)\right)_{k}$. In fact $\left(H_{*}\left(\mathfrak{u}^{-}\right)\right)_{k}=H_{k}\left(\mathfrak{u}^{-}\right)$and hence

$$
\operatorname{dim}\left(H_{*}\left(\mathfrak{u}^{-}\right)\right)_{k}=(-1)^{k} b_{k}
$$

Proof. One has $\left(H_{n}\left(\mathfrak{u}^{-}\right)\right)_{k}=0$ unless $n=k$ by (4.28) and Theorem 4.21. But (4.28) implies that $\left(\operatorname{Har}_{k}\left(\wedge \mathfrak{u}^{-}\right)\right)_{k}$ is, as a $\mathfrak{g}$-module, given by the direct sum $\sum_{\xi \in \Xi_{k}} V_{\xi}$ 
(using Theorems 4.5 and 4.28). The remaining statements follow from Theorem 4.23. QED

4.7. For any complex number $s$ one can define $s$ power of Euler product $\prod_{n=1}^{\infty}(1-$ $x^{n}$ ) by taking the logarithm of the Euler product, multiplying by $s$ and then exponeniating. It follows easily that

$$
\left(\prod_{n=1}^{\infty}\left(1-x^{n}\right)\right)^{s}=\sum_{k=0}^{\infty} f_{k}(s) x^{k}
$$

where $f_{k}(s)$ is a polynomial of degree $k$ defined as follows: Let $\mu: \mathbb{N} \rightarrow \mathbb{Q}$ be defined by putting $\mu(m)=\sum_{d \mid m} 1 / d$. For $k, n \in \mathbb{N}, n \leq k$, let

$$
Q_{k, n}=\left\{q \in \mathbb{N}^{n} \mid q=\left(m_{1}, \ldots, m_{n}\right), \sum_{i=1}^{n} m_{i}=k\right\}
$$

and using this notation let

$$
q_{k, n}=\sum_{q \in Q_{k, n}} \mu\left(m_{1}\right) \cdots \mu\left(m_{n}\right)
$$

Put $f_{0}=1$. If $k \in \mathbb{N}$ let $f_{k}(s)$ be the polynomial of degree $k$ (with 0 constant term ) defined by putting

$$
f_{k}(s)=\sum_{n=1}^{k} q_{k, n}(-s)^{n} / n !
$$

Of course this is a very complicated expression for $f_{k}(s)$. In the notation of Theorem 4.23 one has

$$
b_{k}=f_{k}(\operatorname{dim} \mathfrak{k})
$$

Clearly the polynomial $f_{k}(s)$ would be known if we knew its roots.

Remark 4.25. According to Serre (see top of p. 98 in [Se]) it is a question raised by D. H. Lehmer as to whether 24 is ever a root of $f_{k}(s)$ for any $k \in \mathbb{Z}_{+}$.

It is easy to see that $f_{1}(s)=-s$ and that in fact 0 is a root of $f_{k}(s)$ for any $k \in \mathbb{N}$. We will determine $f_{2}(s), f_{3}(s)$ and $f_{4}(s)$ in a uniform way using Theorem 4.23 . We first observe

Proposition 4.26. For the missing roots (to be determined in Theorem 4.27 below) $r_{4}, r_{3}$ and $r_{2}$ one has

$$
\begin{aligned}
f_{4}(s) & =1 / 4 ! s(s-1)(s-3)\left(s-r_{4}\right) \\
-f_{3}(s) & =1 / 3 ! s(s-1)\left(s-r_{3}\right) \\
f_{2}(s) & =1 / 2 ! s\left(s-r_{2}\right)
\end{aligned}
$$


Proof. Euler has determined the right side of (4.67) when $s=1$. The only nonzero coefficients on the right side of (4.67) are the coefficients of the pentagonal powers $x^{\left(3 n^{2}-n\right) / 2}$ where $n \in \mathbb{Z}$. Since 3 and 4 are not pentagonal numbers it follows that 1 must be a root of $f_{3}(s)$ and $f_{4}(s)$. Now Jacobi has determined the right side of (4.67) when $s=3$. Here the only nonzero coefficients on the right side of (4.67) are the coefficients of the triangular powers $x^{n(n+1) / 2}$ for $n \in \mathbb{Z}_{+}$. Since 4 is not a triangular number, 3 must be a root of $f_{4}(s)$. This proves the proposition. QED

Malcev has determined $M$ (see $§ 4.1$ ) for all complex simple Lie algebras (see $\S 4.3$ in [Ko-2]). There are only 3 cases where $M<h^{\vee}$, namely the cases where $\mathfrak{g}$ is of $A_{1}, A_{2}$ and $G_{2}$. The relevant information is in the following table.

$\begin{array}{ccccc}\frac{\mathfrak{g} \text { type }}{A_{1}} & & \frac{M}{1} & \frac{h^{\vee}}{2} & \frac{\text { dim } \mathfrak{k}}{3} \\ A_{2} & 2 & 3 & 8 \\ G_{2} & 3 & 4 & 14\end{array}$

But then, by Theorem $4.23, C_{4}=0$ and hence $b_{4}=0$ if $\mathfrak{g}$ is of type $G_{2}$. Next $C_{3}=0$ and hence $b_{3}=0$ if $\mathfrak{g}$ is of type $A_{2}$. Finally $C_{2}=0$ and hence $b_{2}=0$ if $\mathfrak{g}$ is of type $A_{1}$. For these three cases $f_{h^{\vee}}(\operatorname{dim} \mathfrak{k})=0$ by (4.69). Hence we have proved

Theorem 4.27. The missing roots $r_{4}, r_{3}$ and $r_{2}$ in Proposition 4.26 are, respectively, the complex dimensions of $G_{2}, A_{2}$ and $A_{1}$, namely 14,8 and 3 so that

$$
\begin{aligned}
f_{4}(s) & =1 / 4 ! s(s-1)(s-3)(s-14) \\
-f_{3}(s) & =1 / 3 ! s(s-1)(s-8) \\
f_{2}(s) & =1 / 2 ! s(s-3)
\end{aligned}
$$

Let $k$ be any positive integer. If $m \in \mathbb{Z}_{+}$and $m \geq 2$ let $C_{k}(m)$ equal $C_{k}$ for the case where $K=S U(m)$. If $m \geq k$ then $k \leq h^{\vee}=m$ and hence, by Theorem 4.23,

$$
f_{k}\left(m^{2}-1\right)=(-1)^{k} \operatorname{dim} C_{k}(m)
$$

In particular note that $f_{k}\left(m^{2}-1\right) \neq 0$ since $M \geq k$ where $M$ is defined here for $K=S U(m)$. But since $f_{k}(0)=0$ and since $f_{k}$ is a polynomial of degree $k$ it follows that $f_{k}(s)$ is determined by the values $f_{k}\left(m^{2}-1\right)$ for $k$ different positive values of $m$. But then (4.70) establishes the following theorem. The result implies that $f_{k}(s)$ is encoded in the $k$-dimensional commutative subalgebra structure of $\operatorname{Lie} S l(m, \mathbb{C})$ for $k$ different values of $m$ where $m \geq k$ and $m>1$.

Theorem 4.28. Let $k$ be a positive integer. Then $f_{k}(s)$ is determined by the numbers $\operatorname{dim} C_{k}(m)$ for $k$ different values of $m \in \mathbb{Z}_{+}$where $m \geq k$ and $m>1$. Furthermore under the assumption $m \in \mathbb{Z}_{+}, m \geq k$ and $m>1$ one has $f_{k}\left(m^{2}-1\right) \neq$ 0 . 


\section{References}

[A-F] R. Adin and A. Frumkin, Rim Hook tableau and Kostant's $\eta$-Function Coefficients, arXiv:math. CO/0201003 v2

[B] R. Bott, An Application of the Morse Theory to the Topology of Lie Groups, Bull. Soc. Math. France, 84(1956), 251-281

[C-P] P. Cellini and P. Papi, ad-Nilpotent Ideals of a Borel Subalgebra, J. Alg., 225(2000), 130-141

[F] H. D. Fegan, The heat equation and modular forms, J. Diff. Geom., 13(1978), 589-602

[G] H. Garland, Dedekind's $\eta$-function and the cohomology of infinite dimensional Lie algebras, PNAS, USA, 72(1975), 2493-2495

[G-L] H. Garland and J. Lepowsky, Lie algebra homology and the Macdonald-Kac formulas, Inventiones Math., 34(1976), 37-76

[Ka] V. Kac, Infinite dimensional Lie algebras, Cambridge 1990

[Ko-1] B.Kostant, Lie algebra cohomology and the generalized Borel-Weil theorem, Ann. of Math., 74 No. 2. (1961), 329-387

[Ko-2] B. Kostant, Eigenvalues of a Laplacian and Commutative Lie subalgebras, Topology, 3(1965), 147-159

[Ko-3] B. Kostant, On Macdonald's $\eta$-function Formula, the Lapalacian and Generalized Exponents, Adv. in Math. 20 No.2. (1976), 179-212

[Ko-4] B. Kostant, The McKay correspondence, the Coxeter element and representation theory, Société Mathématique de France, Astérisque, hors série, 1985, 209-255

[Ko-5] B. Kostant, The set of Abelian ideals of a Borel Subalgebra, Cartan decompositions and Discrete Series Representations, IMRN, 5(1998), 225-252

[Ko-6] B. Kostant, On $\wedge \mathfrak{g}$ for a Semisimple Lie Algebra $\mathfrak{g}$, as an Equivariant Module over the Symmetric Algebra $\mathrm{S}(\mathrm{g})$, Advanced Studies in Pure Mathematics, 26(2000), Analysis on Homogeneous Spaces and Representation Theory of Lie Groups, 129-144

[Ku-1] S. Kumar, Geometry of Schubert cells and cohomology of Kac-moody Lie algebras, J. Diff. Geom., 20(1984), 389-431

[Ku-2] S. Kumar, Kac-Moody Groups, their Flag Varities and Representation Theory, PM 204, Birkhauser Boston, 2002

[L] D. H. Lehmer, Ramanujan's function $\tau(n)$, Duke Math J., 30(1943)

[Ma-1] I.G. MacDonald, Affine root systems and Dedekind's $\eta$-function, Invent. Math. 15(1972), 91-143

[Ma-2] I. G. Macdonald, Symmetric functions and Hall Polynomials, Oxford 1995, (2nd Edition)

[Se] J-P. Serre, A Course in Arithmetic, GTM, 7 Springer-Verlag, 1973

[St] R. P. Stanley, Enumerative Combinatorics, 2, Cambridge, 1999

[Su] R. Suter, Abelian ideals in a Borel subalgebra of a complex simple Lie algebra, arXiv:math.RT/0210463 v1, 30 Oct 2002 
[Ze] S. Zelditch, Macdonald's Identities and the Large N Limit of $Y M_{2}$ on the Cylinder, to appear, Comm. Math. Physics

Bertram Kostant

Dept. of Math.

MIT

Cambridge, MA 02139

E-mail kostant@math.mit.edu 